\title{
Structure constants of defect changing operators on the 1/2 BPS Wilson loop
}

\author{
Minkyoo Kim, ${ }^{a, b}$ Naoki Kiryu, ${ }^{c}$ Shota Komatsu ${ }^{d, e}$ and Takuya Nishimura ${ }^{c}$ \\ ${ }^{a}$ National Institute for Theoretical Physics, \\ School of Physics and Mandelstam Institute for Theoretical Physics, \\ University of the Witwatersrand, Johannesburg Wits 2050, South Africa \\ ${ }^{b}$ MTA Lendulet Holographic QFT Group, Wigner Research Centre, \\ Budapest 114 , P.O.B. 49, Hungary \\ ${ }^{c}$ Institute of Physics, University of Tokyo, Komaba, Meguro-ku, \\ Tokyo 153-8902 Japan \\ ${ }^{d}$ Perimeter Institute for Theoretical Physics, \\ Waterloo, Ontario N2L 2Y5, Canada \\ e School of Natural Sciences, Institute for Advanced Study, \\ Princeton, New Jersey 08540, U.S.A. \\ E-mail: minkyoo.kim@wits.ac.za, kiryu@hep1.c.u-tokyo.ac.jp, \\ shota.komadze@gmail.com, tnishimura@hep1.c.u-tokyo.ac.jp
}

ABSTRACT: We study three-point functions of operators on the 1/2 BPS Wilson loop in planar $\mathcal{N}=4$ super Yang-Mills theory. The operators we consider are "defect changing operators", which change the scalar coupled to the Wilson loop. We first perform the computation at two loops in general set-ups, and then study a special scaling limit called the ladders limit, in which the spectrum is known to be described by a quantum mechanics with the $\operatorname{SL}(2, \mathbb{R})$ symmetry. In this limit, we resum the Feynman diagrams using the Schwinger-Dyson equation and determine the structure constants at all order in the rescaled coupling constant. Besides providing an interesting solvable example of defect conformal field theories, our result gives invaluable data for the integrability-based approach to the structure constants.

KEywords: 1/N Expansion, Conformal Field Theory, AdS-CFT Correspondence, Integrable Field Theories

ARXIV EPRINT: 1710.07325 


\section{Contents}

1 Introduction 2

2 Set-up and conventions 3

2.1 Set-up for three-point functions 3

2.2 Weak coupling expansion 4

2.3 Action and propagators 5

3 Two-point functions at two loops $\quad 6$

3.1 One loop 6

$\begin{array}{lll}3.2 & \text { Two loops } & 7\end{array}$

4 Three-point functions at two loops $\quad 12$

$\begin{array}{lll}4.1 \text { One loop } & 12\end{array}$

$\begin{array}{lll}4.2 & \text { Two loops } & 13\end{array}$

5 Three-point functions in the ladders limit $\quad 14$

$\begin{array}{lll}5.1 \text { Set up } & 15\end{array}$

5.2 Four-point kernel and the Schwinger-Dyson equation 17

$\begin{array}{lll}5.3 & \text { Two-point functions and renormalization } & 19\end{array}$

5.4 Case I: 1 nontrivial and 2 trivial DCOs 21

5.5 Case II: 2 nontrivial and 1 trivial DCOs 22

5.6 Case III: 3 nontrivial DCOs 24

$\begin{array}{llr}6 & \text { Discussion } & 27\end{array}$

$\begin{array}{lr}\text { A Basic integrals } & 29\end{array}$

B Vertex and self-energy diagrams for the three-point functions 30

$\begin{array}{ll}\text { C Excited states and conformal descendants } & 30\end{array}$

D Contribution from the integral of $\Gamma_{\mathrm{UV}} \quad 33$

$\begin{array}{ll}\text { E Evaluation of the integral (5.35) } & \mathbf{3 4}\end{array}$

F An infinite sum representation for $C_{\bullet \bullet} \quad 35$ 


\section{Introduction}

Nonlocal operators and defects enrich our knowledge of interacting quantum field theories: in theories with mass gap, they can be important order parameters which help to distinguish different phases. The prototypical examples of such operators are the Wilson and the 't Hooft loops in gauge theories. In conformal field theories on the other hand, conformal defects lead to a new class of crossing equations, which constrain both operators in the bulk and operators on the defect $[1,2]$.

The main focus of this paper is a theory which is a gauge theory and also a conformal field theory; namely $\mathcal{N}=4$ super Yang-Mills theory $(\mathcal{N}=4 \mathrm{SYM})$. We in particular study the three-point function of defect changing operators on the 1/2 BPS Wilson loop.

The $1 / 2$ BPS Wilson loop is a supersymmetric extension of the ordinary Wilson loop, which is coupled to a scalar as well as to a gauge field, ${ }^{1}$

$$
W \equiv \operatorname{Tr}\left[\operatorname{Pexp}\left(\oint d \tau i A_{\mu} \dot{x}^{\mu}+\phi_{i} n^{i}\left|\dot{x}^{\mu}\right|\right)\right]
$$

Here $n^{i}$ is a six-dimensional unit vector, to be called the $R$-symmetry polarization, which designates the scalar coupled to the Wilson loop. Local operators on the loop (denoted by $\mathcal{O})$ can be introduced by inserting fields inside the trace e.g.

$$
W[\mathcal{O}] \equiv \operatorname{Tr}[\operatorname{Pexp}\left(\int_{-\infty}^{\tau} d \tau^{\prime} i A_{\mu} \dot{x}^{\mu}+\phi_{i} n^{i}\left|\dot{x}^{\mu}\right|\right) \underbrace{Z^{L}}_{\mathcal{O}} \operatorname{Pexp}\left(\int_{\tau}^{\infty} d \tau^{\prime \prime} i A_{\mu} \dot{x}^{\mu}+\phi_{i} \tilde{n}^{i}\left|\dot{x}^{\mu}\right|\right)],
$$

where $Z$ is a complex scalar field. As indicated, the polarization $n^{i}$ can change across the operator insertion. The simplest possible operator among them is the one which has no field insertions and merely changes the polarization. We call such operators the defect changing operators (DCO).

The spectrum of such operators in the planar limit was studied extensively using integrability [3-5]. A natural next step in this direction is to compute their three-point functions. ${ }^{2}$ For ordinary gauge invariant operators, there exists a nonperturbative framework to study the three-point function [8]. It is based on the fact that, in the AdS/CFT correspondence, the three-point function is dual to a closed-string world sheet whose topology is a pair of pants. The key idea in this approach is to decompose such a pair of pants into two hexagons and determine the contribution from each hexagon using integrability.

It is then interesting to ask if we can extend this approach to more general observables involving open strings. The three-point function on the Wilson loop, which we discuss in this paper, is precisely such an observable; it corresponds to the interaction process of three open strings in AdS. With an eye toward such a direction, we perform two perturbative computations in this paper: after summarizing the set-ups and conventions in section 2, we

\footnotetext{
${ }^{1}$ Pexp denotes a path-ordered exponential.

${ }^{2}$ Recently several structure constants on the $1 / 2$ BPS Wilson loop were computed in [6] by taking the limit of generic smooth Wilson loops. Also, the four-point functions of single-letter operators were computed at strong coupling using the Witten diagrams in [7].
} 
first compute the two-point functions of general DCO's at two loops in section 3. Through this computation, we reproduce the anomalous dimensions computed previously in the literature. Furthermore, we also determine the normalizations of the operators, which are prerequisite for computing the scheme-independent structure constants. We then compute the three-point function at two loops in section 4. After doing so, we focus on a special scaling limit called the ladders limit [9] in section 5, and compute the structure constants at all orders in the rescaled coupling constant using the Schwinger-Dyson equation. These results would provide important datapoints for developing the integrability-based approach in the future. In section 6 , we conclude and comment on the prospects. A few appendices are included to elucidate technical details.

Note added: while we were writing up this article, we became aware that A. Cavaglia, N. Gromov, and F. Levkovich-Maslyuk were working on a similar topic and obtained independently the results that overlap the contents of this paper. We thank them for informing us of their upcoming paper [10].

\section{Set-up and conventions}

\subsection{Set-up for three-point functions}

Correlation functions on the $1 / 2$ BPS straight-line Wilson loop are constrained by the $\mathrm{SL}(2, \mathbb{R})$ symmetry preserved by the Wilson loop [11]. This in particular implies that the space-time dependences of the two- and the three-point functions are completely determined. Namely, we have

$$
\begin{aligned}
\left\langle W\left[\mathcal{O}_{1}\left(t_{1}\right) \mathcal{O}_{2}\left(t_{2}\right)\right]\right\rangle & =\frac{\delta_{12}}{\left|t_{12}\right|^{2 \Delta_{1}}}, \\
\left\langle W\left[\mathcal{O}_{1}\left(t_{1}\right) \mathcal{O}_{2}\left(t_{2}\right) \mathcal{O}_{3}\left(t_{3}\right)\right]\right\rangle & =\frac{C_{123}}{\left|t_{12}\right|^{\Delta_{1}+\Delta_{2}-\Delta_{3}}\left|t_{23}\right|^{\Delta_{2}+\Delta_{3}-\Delta_{1}}\left|t_{31}\right|^{\Delta_{3}+\Delta_{1}-\Delta_{2}}},
\end{aligned}
$$

where $t_{i}$ 's are positions of the operators and $t_{i j} \equiv t_{i}-t_{j} . \Delta_{i}$ and $C_{123}$ are the conformal dimension and the structure constant respectively.

As mentioned in the introduction, the main focus of this paper is the structure constant of the defect changing operators. The most general three-point functions of such operators are characterized by three six-dimensional unit vectors $n_{i j}$ parametrizing the directions of the scalars coupled to each segment of the Wilson loop (see figure 1):

$$
\begin{aligned}
& \left\langle W\left[\mathcal{O}_{1}\left(t_{1}\right) \mathcal{O}_{2}\left(t_{2}\right) \mathcal{O}_{3}\left(t_{3}\right)\right]\right\rangle \\
& \equiv\left\langle\operatorname { T r } \left[\operatorname{Pexp}\left(\int_{-\infty}^{t_{1}} d \tau i A_{\mu} \dot{x}^{\mu}+\phi_{i} n_{31}^{i}\left|\dot{x}^{\mu}\right|\right) \operatorname{Pexp}\left(\int_{t_{1}}^{t_{2}} d \tau i A_{\mu} \dot{x}^{\mu}+\phi_{i} n_{12}^{i}\left|\dot{x}^{\mu}\right|\right)\right.\right. \\
& \left.\left.\quad \operatorname{Pexp}\left(\int_{t_{2}}^{t_{3}} d \tau i A_{\mu} \dot{x}^{\mu}+\phi_{i} n_{23}^{i}\left|\dot{x}^{\mu}\right|\right) \operatorname{Pexp}\left(\int_{t_{3}}^{\infty} d \tau i A_{\mu} \dot{x}^{\mu}+\phi_{i} n_{31}^{i}\left|\dot{x}^{\mu}\right|\right)\right]\right\rangle .
\end{aligned}
$$

It is often useful to parametrize the scalar couplings by the angles between vectors $n_{i j}$ as

$$
\cos \theta_{1} \equiv n_{31} \cdot n_{12}, \quad \cos \theta_{2} \equiv n_{12} \cdot n_{23}, \quad \cos \theta_{3} \equiv n_{23} \cdot n_{31} .
$$




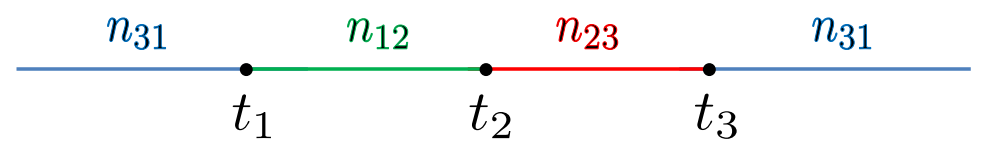

Figure 1. Three-point function of defect changing operators. Each segment is coupled to a different scalar specified by the polarizations $n_{i j}$ 's.

\subsection{Weak coupling expansion}

The structures of the two- and three-point functions given in (2.1) apply only to properly renormalized operators. However, in the actual computation at weak coupling, we often study the correlators of un-renormalized (or equivalently bare) operators. It is therefore useful to know how to extract the conformal data from such un-renormalized correlators.

Let us first analyze the two-point functions. In general, the bare operator $\mathcal{O}^{B}$ and the renormalized operator $\mathcal{O}^{R}$ are related $\mathrm{as}^{3}$

$$
\mathcal{O}^{R} \equiv \frac{\epsilon^{-\gamma}}{\sqrt{a}} \mathcal{O}^{B}
$$

where $\epsilon \sim \Lambda^{-1}$ is the cut-off, $\gamma$ is the anomalous dimension and $a$ is the finite renormalization constant needed to bring the renormalized correlator into a canonical form (2.1). Substituting (2.4) to (2.1), we can determine the structure of the un-renormalized two-point function as

$$
\left\langle W\left[\mathcal{O}^{B}\left(t_{1}\right) \mathcal{O}^{B}\left(t_{2}\right)\right]\right\rangle=\frac{a}{\left|t_{12}\right|^{2 \Delta^{(0)}}} \frac{1}{\left(\left|t_{12}\right| / \epsilon\right)^{2 \gamma}},
$$

where $\Delta^{(0)}$ is the bare dimension. Both $\gamma$ and $a$ are functions of the 't Hooft coupling constant $\lambda \equiv g_{\mathrm{YM}}^{2} N$, and can be expanded as

$$
a=1+\lambda a^{(1)}+\lambda^{2} a^{(2)}+\cdots, \quad \gamma=\lambda \gamma^{(1)}+\lambda^{2} \gamma^{(2)}+\cdots .
$$

Here we assumed that the correlator is correctly normalized at tree level, namely $\left.a\right|_{\lambda=0}=1$. By expanding the right hand side of (2.5), we obtain the expression at weak coupling,

$$
\left\langle W\left[\mathcal{O}^{B}\left(t_{1}\right) \mathcal{O}^{B}\left(t_{2}\right)\right]\right\rangle=\frac{\left(1+\lambda A^{(1)}+\lambda^{2} A^{(2)}+\cdots\right)}{\left|t_{12}\right|^{2 \Delta_{1}^{(0)}}},
$$

with

$$
\begin{aligned}
& A^{(1)}=a^{(1)}-2 \gamma^{(1)} \log \frac{\left|t_{12}\right|}{\epsilon}, \\
& A^{(2)}=a^{(2)}-2 a^{(1)} \gamma^{(1)} \log \frac{\left|t_{12}\right|}{\epsilon}+2\left(\gamma^{(1)} \log \frac{\left|t_{12}\right|}{\epsilon}\right)^{2}-2 \gamma^{(2)} \log \frac{\left|t_{12}\right|}{\epsilon} .
\end{aligned}
$$

From the relation (2.4) and the structure of the renormalized three-point function (2.1), we can also determine the structure of the bare three-point functions at weak coupling. To

\footnotetext{
${ }^{3}$ Here we ignored the operator mixing since it never appears in the problems studied in this paper.
} 
simplify the expression, below we set $a^{(1)}=0$ as it is satisfied in all the examples studied in this paper. Then, using the expansion of the structure constant,

$$
C_{123}=C_{123}^{(0)}\left(1+\lambda c_{123}^{(1)}+\lambda^{2} c_{123}^{(2)}+\cdots\right),
$$

one can write the result as

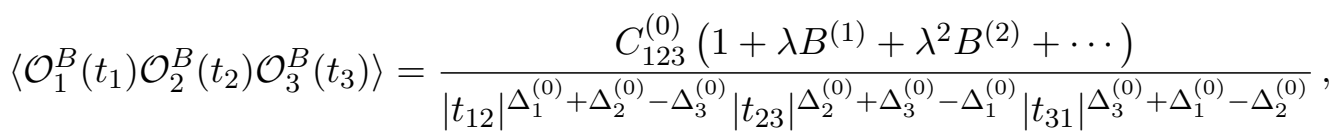

with

$$
\begin{aligned}
B^{(1)} & =c_{123}^{(1)}-\sum_{i} \gamma_{i}^{(1)} \log u_{i}, \\
B^{(2)} & =c_{123}^{(2)}+\frac{1}{2} \sum_{i} a_{i}^{(2)}-\sum_{i}\left(\gamma_{i}^{(2)}+c_{123}^{(1)} \gamma_{i}^{(1)}\right) \log u_{i}+\frac{1}{2} \sum_{i, j} \gamma_{i}^{(1)} \gamma_{j}^{(1)} \log u_{i} \log u_{j} .
\end{aligned}
$$

Here $a_{i}$ and $\gamma_{i}$ are the normalization and the anomalous dimension of the operator $\mathcal{O}_{i}$ respectively and $u_{i}$ is given by

$$
u_{i} \equiv\left|\frac{t_{i j} t_{k i}}{t_{j k} \epsilon}\right|
$$

where $\{i, j, k\}$ is a cyclic permutation of $\{1,2,3\}$.

In the following sections, we will compare the results from perturbation with the expressions (2.7) and (2.10) and read off the anomalous dimension and the structure constant.

\subsection{Action and propagators}

The Euclidean action ${ }^{4}$ of $\mathcal{N}=4 \mathrm{SYM}$ used in this paper is

$$
\begin{aligned}
S & =\frac{1}{g_{\mathrm{YM}}^{2}} \int d^{4} x \mathcal{L}, \\
\mathcal{L} & =\operatorname{Tr}\left[-\frac{\left[D_{\mu}, D_{\nu}\right]^{2}}{2}+\left(D_{\mu} \phi_{i}\right)^{2}+\frac{\left[\phi_{i}, \phi_{j}\right]^{2}}{2}+i \bar{\psi} \Gamma^{\mu} D_{\mu} \psi\right. \\
& \left.+\bar{\psi} \Gamma^{i}\left[\phi_{i}, \psi\right]+\partial^{\mu} \bar{c} D_{\mu} c+\left(\partial_{\mu} A^{\mu}\right)^{2}\right],
\end{aligned}
$$

with $D_{\mu} \equiv \partial_{\mu}-i\left[A_{\mu}, \quad\right]$. Here $c$ and $\bar{c}$ are the ghosts and $\Gamma^{A}=\left(\Gamma^{\mu}, \Gamma^{i}\right)$ are the tendimensional Dirac matrices satisfying

$$
\operatorname{tr}\left(\Gamma^{A} \Gamma^{B}\right)=16 \delta^{A B} .
$$

To emphasize that the trace here is not over the $\mathrm{SU}(N)$ colour degrees of freedom, here we used the lowercase letters, tr.

\footnotetext{
${ }^{4}$ Our convention is essentially the same as (42) in [12]. The differences are

1. We write the action in terms of traces instead of decomposing the fields into the generators of $\mathrm{SU}(N)$.

2. We chose the Feynman gauge by setting $\xi$ in (42) of [12] to be 1 .

3. A sign error in front of the scalar quartic interaction in [12] was corrected.
} 


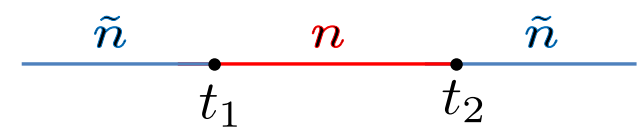

Figure 2. Two-point function of defect changing operators.

Using this action, one can compute the propagators as follows:

$$
\begin{aligned}
& \text { Gluon : } \mu \underset{b}{\stackrel{a}{\sim} \sim \sim_{d}^{c} \nu} \nu=\frac{g_{\mathrm{YM}}^{2} \delta^{a c} \delta^{b d}}{8 \pi^{2}} \frac{\delta_{\mu \nu}}{|x-y|^{2}} \text {, } \\
& \text { Scalar : } i \frac{a}{b} \quad \frac{c}{d} j=\frac{g_{\mathrm{YM}}^{2} \delta^{a c} \delta^{b d}}{8 \pi^{2}} \frac{\delta_{i j}}{|x-y|^{2}} \text {, } \\
& \text { Fermion : } \begin{array}{c}
a \\
-b
\end{array} \quad=\frac{g_{\mathrm{YM}}^{2} \delta^{a c} \delta^{b d}}{8 \pi^{2}} \frac{1}{|x-y|^{2}}, \\
& \text { Ghost : } \frac{a}{b}-\frac{c}{d}=\frac{g_{\mathrm{YM}}^{2} \delta^{a c} \delta^{b d}}{8 \pi^{2}} \frac{1}{|x-y|^{2}} \text {, }
\end{aligned}
$$

Here $a$ - $d$ are the color indices and all the propagators are proportional to $\delta^{a c} \delta^{b d}$. To compute the loop corrections, one just need to bring down the interaction terms by expanding $e^{-S}$ and Wick-contract the fields using the propagators.

\section{Two-point functions at two loops}

Let us first compute the two-point function of defect changing operators at two loops. The purpose of this section is twofold; to reproduce the anomalous dimension known in the literature and to determine the normalization of the operator, which is necessary for extracting the scheme-independent structure constant from perturbative three-point functions.

\subsection{One loop}

The two-point function we compute is of the following form (see also figure 2):

$$
\begin{aligned}
&\left\langle W\left[\mathcal{O}_{1}\left(t_{1}\right) \mathcal{O}_{2}\left(t_{2}\right)\right]\right\rangle \equiv\left\langle\operatorname { T r } \left[\operatorname{Pexp}\left(\int_{-\infty}^{t_{1}} d \tau i A_{\mu} \dot{x}^{\mu}+\phi_{i} \tilde{n}^{i}\left|\dot{x}^{\mu}\right|\right)\right.\right. \\
&\left.\left.\operatorname{Pexp}\left(\int_{t_{1}}^{t_{2}} d \tau i A_{\mu} \dot{x}^{\mu}+\phi_{i} n^{i}\left|\dot{x}^{\mu}\right|\right) \operatorname{Pexp}\left(\int_{t_{2}}^{\infty} d \tau i A_{\mu} \dot{x}^{\mu}+\phi_{i} \tilde{n}^{i}\left|\dot{x}^{\mu}\right|\right)\right]\right\rangle .
\end{aligned}
$$

Owing to the $\mathrm{SO}(6)$ invariance, it only depends on the inner product $n \cdot \tilde{n}$ and the coupling constant $\lambda$. To perform the perturbative computation, we just need to expand the exponentials in (3.1) and contract them with propagators and vertices.

At one loop, one can only have a single propagator (without vertices). The propagator can be either a gauge field or a scalar field and takes the form,

$$
\text { Gauge : }-\frac{\lambda}{8 \pi^{2}} \frac{1}{\tau_{12}^{2}}, \quad \text { Scalar }:\left(n_{1} \cdot n_{2}\right) \frac{\lambda}{8 \pi^{2}} \frac{1}{\tau_{12}^{2}},
$$



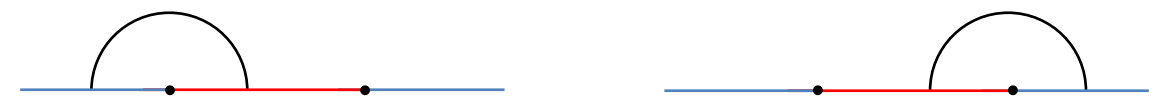

Figure 3. One-loop corrections to the two-point function of defect changing operators. Owing to the cancellation between gluons and scalars, the only diagrams that survive are the ones in which the propagator connects two different segments.

where $\tau_{12}=\tau_{1}-\tau_{2}$ is the distance between two end points and $n_{1}$ and $n_{2}$ (which can be either $n$ or $\tilde{n}$ ) are the polarization vectors at each end point. The extra minus sign for gluons comes from factors of $i$ in the exponentials in (3.1). As is clear from (3.2), the contributions from the gauge field and the scalar field cancel out if $n_{1}=n_{2}$, since both $n$ and $\tilde{n}$ have a unit norm. We are thus left with diagrams in which a propagator connects two segments with different polarizations (see figure 3).

To compute such diagrams, we introduce a UV regularization by cutting out a small circle of radius ${ }^{5} \epsilon / 2$ around each operator. This leads to a regularized integral

$$
\left\langle W\left[\mathcal{O}_{1}\left(t_{1}\right) \mathcal{O}_{2}\left(t_{2}\right)\right]\right\rangle_{1-\text { loop }}=\frac{\lambda(n \cdot \tilde{n}-1)}{8 \pi^{2}}\left(\int_{-\infty}^{t_{1}^{-}} d \tau_{1} \int_{t_{1}^{+}}^{t_{2}^{-}} d \tau_{2} \frac{1}{\tau_{12}^{2}}+\int_{t_{1}^{+}}^{t_{2}^{-}} d \tau_{1} \int_{t_{2}^{+}}^{\infty} d \tau_{2} \frac{1}{\tau_{12}^{2}}\right),
$$

with $t_{i}^{ \pm}=t_{i} \pm \epsilon / 2$. This integral can be easily evaluated using

$$
\int_{d}^{c} d \tau_{1} \int_{b}^{a} d \tau_{2} \frac{1}{\tau_{12}^{2}}=\log \frac{(a-c)(b-d)}{(a-d)(b-c)}
$$

as

$$
\left\langle W\left[\mathcal{O}_{1}\left(t_{1}\right) \mathcal{O}_{2}\left(t_{2}\right)\right]\right\rangle_{1-\operatorname{loop}}=\frac{\lambda(n \cdot \tilde{n}-1)}{4 \pi^{2}} \log \frac{t_{12}}{\epsilon}+O(\epsilon) .
$$

Comparing this with (2.7), we can determine the one-loop normalization $a^{(1)}$ and the anomalous dimension $\gamma^{(1)}$ as

$$
a^{(1)}=0, \quad \gamma^{(1)}=\frac{1-n \cdot \tilde{n}}{8 \pi^{2}} .
$$

The result for $\gamma^{(1)}$ of course matches the one in the literature [13], but it also shows that the normalization at one loop $a^{(1)}$ vanishes in our scheme.

\subsection{Two loops}

Let us now proceed to the two-loop computation. At two loops, there appear three types of diagrams; the ladder, the vertex and the self-energy. Below we are going to evaluate them one by one.

Ladder diagrams. The first diagrams are the ladder diagrams, which consist only of propagators. For this class of diagrams, the computation proceeds in a similar manner as in the previous subsection. Also here, the diagrams that contain propagators connecting the same segment vanish due to the cancellation between the scalar and the gluon.

\footnotetext{
${ }^{5}$ The factor of $1 / 2$ is just a useful convention which simplifies the final result.
} 


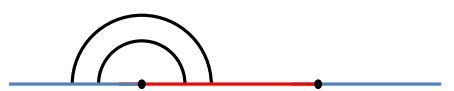

L1

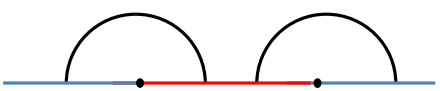

L2

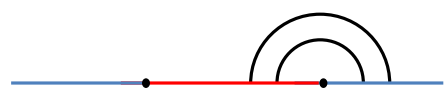

L3

Figure 4. Ladder diagrams that contribute to the two-point function at two loops. Here thick black curves represent either a scalar propagator or a gluon propagator.

Thus only non-zero diagrams are the ones depicted in figure 4, which are given by

$$
\begin{aligned}
& \mathrm{L} 1=\left(\frac{(n \cdot \tilde{n}-1) \lambda}{8 \pi^{2}}\right)^{2} \int_{-\infty}^{t_{1}^{-}} d \tau_{1} \int_{\tau_{1}}^{t_{1}^{-}} d \tau_{2} \int_{t_{1}^{+}}^{t_{2}^{-}} d \tau_{3} \int_{\tau_{3}}^{t_{2}^{-}} d \tau_{4} \frac{1}{\tau_{14}^{2}} \frac{1}{\tau_{23}^{2}}, \\
& \mathrm{~L} 2=\left(\frac{(n \cdot \tilde{n}-1) \lambda}{8 \pi^{2}}\right)^{2} \int_{-\infty}^{t_{1}^{-}} d \tau_{1} \int_{t_{1}^{+}}^{t_{2}^{-}} d \tau_{2} \int_{\tau_{2}}^{t_{2}^{-}} d \tau_{3} \int_{t_{2}^{+}}^{\infty} d \tau_{4} \frac{1}{\tau_{12}^{2}} \frac{1}{\tau_{34}^{2}}, \\
& \mathrm{~L} 3=\left(\frac{(n \cdot \tilde{n}-1) \lambda}{8 \pi^{2}}\right)^{2} \int_{t_{1}^{+}}^{t_{2}^{-}} d \tau_{1} \int_{\tau_{1}}^{t_{2}^{-}} d \tau_{2} \int_{t_{2}^{+}}^{\infty} d \tau_{3} \int_{\tau_{3}}^{\infty} d \tau_{4} \frac{1}{\tau_{14}^{2}} \frac{1}{\tau_{23}^{2}} .
\end{aligned}
$$

Each of these integrals can be evaluated straightforwardly as follows: ${ }^{6}$

$$
\begin{aligned}
\mathrm{L} 1=\mathrm{L} 3 & =\left(\frac{(n \cdot \tilde{n}-1) \lambda}{8 \pi^{2}}\right)^{2}\left[\frac{\pi^{2}}{6}-\log \frac{t_{21}}{\epsilon}+\frac{1}{2}\left(\log \frac{t_{21}}{\epsilon}\right)^{2}\right]+O(\epsilon), \\
\mathrm{L} 2 & =\left(\frac{(n \cdot \tilde{n}-1) \lambda}{8 \pi^{2}}\right)^{2}\left[-\frac{\pi^{2}}{6}+\left(\log \frac{t_{21}}{\epsilon}\right)^{2}\right]+O(\epsilon) .
\end{aligned}
$$

Summing three terms, we get

$$
\mathrm{L} \equiv \mathrm{L} 1+\mathrm{L} 2+\mathrm{L} 3=\left(\frac{(n \cdot \tilde{n}-1) \lambda}{8 \pi^{2}}\right)^{2}\left[\frac{\pi^{2}}{6}-2 \log \frac{t_{21}}{\epsilon}+2\left(\log \frac{t_{21}}{\epsilon}\right)^{2}\right] .
$$

Vertex diagrams. The second diagrams are the ones which contain one interaction vertex. Written explicitly, they arise from the Wick contraction of the following terms:

$$
\begin{aligned}
& \frac{i^{3}}{3 !} \int d \tau_{1} d \tau_{2} d \tau_{3}\left\langle\operatorname{Tr} \mathrm{P}\left[\mathcal{A}\left(\tau_{1}\right) \mathcal{A}\left(\tau_{2}\right) \mathcal{A}\left(\tau_{3}\right)\right]\left(\frac{2 i}{g_{\mathrm{YM}}^{2}} \int d^{4} x \operatorname{Tr}\left\{\partial_{\mu} A_{\nu}(x)\left[A_{\mu}(x), A_{\nu}(x)\right]\right\}\right)\right\rangle \\
& +\frac{i}{2 ! 1 !} \int d \tau_{1} d \tau_{2} d \tau_{3}\left\langle\operatorname{Tr} \mathrm{P}\left[\Phi_{1}\left(\tau_{1}\right) \Phi_{2}\left(\tau_{2}\right) \mathcal{A}\left(\tau_{3}\right)\right]\left(\frac{2 i}{g_{\mathrm{YM}}^{2}} \int d^{4} x \operatorname{Tr}\left\{\partial_{\mu} \phi(x)\left[A_{\mu}(x), \phi(x)\right]\right\}\right)\right\rangle .
\end{aligned}
$$

Here $\mathcal{A} \equiv A_{\mu} \dot{x}^{\mu}$ and $\Phi_{i} \equiv\left(\phi \cdot n_{i}\right)|\dot{x}|$ with $n_{1,2}$ being the polarization vectors at each end-point.

The Wick contraction of the above correlator leads to a set of diagrams shown in figure 5. To illustrate how the computation goes, let us focus on the diagram V1. In the

\footnotetext{
${ }^{6}$ The equivalence between L1 and L3 can be shown at the level of integrands by performing the translation $\tau_{i} \rightarrow \tau_{i}-c$ and the reflection $\tau_{i} \rightarrow-\tau_{i}$.
} 


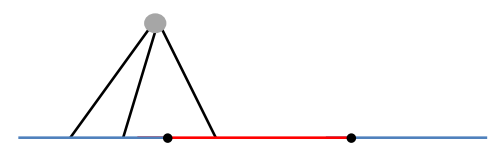

V1

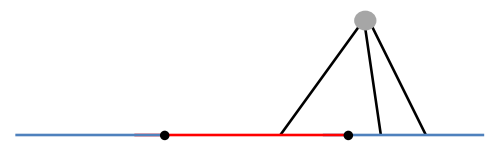

V3

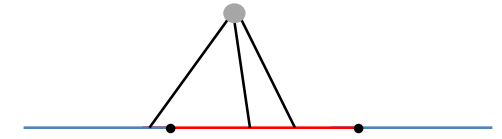

V2

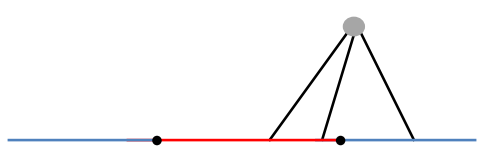

V4

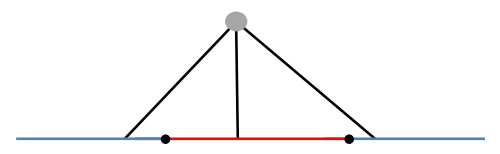

V5

Figure 5. The two-loop diagrams for the two-point function that involve one interaction vertex. Here again, each black line can be either scalar or gluon.

diagram V1, the contribution from the scalar-scalar-gauge vertex consists of three different terms depending on the path-ordering:

$$
\begin{aligned}
i \int_{\substack{\tau_{1}, \tau_{2} \in\left[-\infty, t_{1}\right] \\
\tau_{1}<\tau_{2}}} d \tau_{1} d \tau_{2} \int_{\tau_{3} \in\left[t_{1}, t_{2}\right]} d \tau_{3}\left[\left\langle\operatorname{Tr}\left(\mathcal{A}\left(\tau_{3}\right) \tilde{\Phi}\left(\tau_{2}\right) \tilde{\Phi}\left(\tau_{1}\right)\right)\right\rangle\right. \\
\left.+\left\langle\operatorname{Tr}\left(\Phi\left(\tau_{3}\right) \mathcal{A}\left(\tau_{2}\right) \tilde{\Phi}\left(\tau_{1}\right)\right)\right\rangle+\left\langle\operatorname{Tr}\left(\Phi\left(\tau_{3}\right) \tilde{\Phi}\left(\tau_{2}\right) \mathcal{A}\left(\tau_{1}\right)\right)\right\rangle\right] .
\end{aligned}
$$

Here $\Phi \equiv n \cdot \phi$ and $\tilde{\Phi} \equiv \tilde{n} \cdot \phi$, and we did not write the interaction vertex for brevity. Among these three terms, the first term, which has two scalars in the segment $\left[-\infty, t_{1}\right]$, does not contribute to the final answer since it is proportional to $\tilde{n} \cdot \tilde{n}=1$ and is cancelled precisely by a similar contribution from the three-gauge vertex. On the other hand, from the second term we get

$$
\text { (second term) }=-\frac{\lambda^{2}(n \cdot \tilde{n})}{4\left(4 \pi^{2}\right)^{3}} \int_{\substack{\tau_{1}, \tau_{2} \in\left[-\infty, t_{1}\right] \\ \tau_{1}<\tau_{2}}} d \tau_{1} d \tau_{2} \int_{\tau_{3} \in\left[t_{1}, t_{2}\right]} d \tau_{3}\left(-\partial_{\tau_{1}} Y_{123}+\partial_{\tau_{3}} Y_{123}\right),
$$

with (see appendix A for more details)

$$
Y_{123}\left(\equiv \int \frac{d^{4} x_{5}}{x_{15}^{2} x_{25}^{2} x_{35}^{2}}\right)=-2 \pi^{2}\left(\frac{\log \left|\tau_{12}\right|}{\tau_{13} \tau_{23}}+\frac{\log \left|\tau_{13}\right|}{\tau_{12} \tau_{32}}+\frac{\log \left|\tau_{23}\right|}{\tau_{21} \tau_{31}}\right)
$$

In (3.11), the term $-\partial_{\tau_{1}} Y_{123}$ comes from the contraction with the interaction $\int d^{4} x \operatorname{Tr}\left(\partial_{\mu} \phi A_{\mu} \phi\right)$ while the term $\partial_{\tau_{3}} Y_{123}$ comes from the contraction with $-\int d^{4} x \operatorname{Tr}\left(\partial_{\mu} \phi \phi A_{\mu}\right)$. Similarly the third term (3.10) yields ${ }^{7}$

$$
\text { (third term) }=-\frac{\lambda^{2}(n \cdot \tilde{n})}{4\left(4 \pi^{2}\right)^{3}} \int_{\substack{\tau_{1}, \tau_{2} \in\left[-\infty, t_{1}\right] \\ \tau_{1}<\tau_{2}}} d \tau_{1} d \tau_{2} \int_{\tau_{3} \in\left[t_{1}, t_{2}\right]} d \tau_{3}\left(-\partial_{\tau_{3}} Y_{123}+\partial_{\tau_{2}} Y_{123}\right)
$$

\footnotetext{
${ }^{7}$ Here $-\partial_{\tau_{3}} Y_{123}$ comes from the contraction with $\int d^{4} x \operatorname{Tr}\left(\partial_{\mu} \phi A_{\mu} \phi\right)$ while $\partial_{\tau_{1}} Y_{123}$ comes from the contraction with $-\int d^{4} x \operatorname{Tr}\left(\partial_{\mu} \phi \phi A_{\mu}\right)$.
} 
Adding up the two terms, (3.11) and (3.13), and also the contributions from the three-gauge vertex, we arrive at the following result for the diagram V1:

$$
\mathrm{V} 1=-\frac{\lambda^{2}(n \cdot \tilde{n}-1)}{4\left(4 \pi^{2}\right)^{3}} \int_{-\infty}^{t_{1}^{-}} d \tau_{1} \int_{-\infty}^{t_{1}^{-}} d \tau_{2} \int_{t_{1}^{+}}^{t_{2}^{-}} d \tau_{3} \epsilon\left(\tau_{1}-\tau_{2}\right) \partial_{\tau_{1}} Y_{123} .
$$

Here we used the permutation symmetry of $Y_{123}, Y_{123}=Y_{213}$ etc., to simplify the result and $\epsilon(x) \equiv \theta(x)-\theta(-x)$ with $\theta(x)$ being the step function.

By performing the similar analysis, we arrive at the following results for other diagrams:

$$
\begin{aligned}
& \mathrm{V} 2=-\frac{\lambda^{2}(n \cdot \tilde{n}-1)}{4\left(4 \pi^{2}\right)^{3}} \int_{-\infty}^{t_{1}^{-}} d \tau_{1} \int_{t_{1}^{+}}^{t_{2}^{-}} d \tau_{2} \int_{t_{1}^{+}}^{t_{2}^{-}} d \tau_{3} \epsilon\left(\tau_{2}-\tau_{3}\right) \partial_{\tau_{2}} Y_{123}, \\
& \mathrm{~V} 3=-\frac{\lambda^{2}(n \cdot \tilde{n}-1)}{4\left(4 \pi^{2}\right)^{3}} \int_{t_{1}^{+}}^{t_{2}^{-}} d \tau_{1} \int_{t_{2}^{+}}^{\infty} d \tau_{2} \int_{t_{2}^{+}}^{\infty} d \tau_{3} \epsilon\left(\tau_{2}-\tau_{3}\right) \partial_{\tau_{2}} Y_{123}, \\
& \mathrm{~V} 4=-\frac{\lambda^{2}(n \cdot \tilde{n}-1)}{4\left(4 \pi^{2}\right)^{3}} \int_{t_{1}^{+}}^{t_{2}^{-}} d \tau_{1} \int_{t_{1}^{+}}^{t_{2}^{-}} d \tau_{2} \int_{t_{2}^{+}}^{\infty} d \tau_{3} \epsilon\left(\tau_{1}-\tau_{2}\right) \partial_{\tau_{1}} Y_{123}, \\
& \mathrm{~V} 5=-\frac{\lambda^{2}(n \cdot \tilde{n}-1)}{4\left(4 \pi^{2}\right)^{3}} \int_{-\infty}^{t_{1}^{-}} d \tau_{1} \int_{t_{1}^{+}}^{t_{2}^{-}} d \tau_{2} \int_{t_{2}^{+}}^{\infty} d \tau_{3}\left(\partial_{\tau_{1}} Y_{123}-\partial_{\tau_{3}} Y_{123}\right) .
\end{aligned}
$$

To proceed, we perform the integration by parts to each contribution and rewrite them using $\partial_{x} \epsilon(x)=2 \delta(x)$ as

$$
\begin{aligned}
\mathrm{V} 1 & =-\frac{\lambda^{2}(n \cdot \tilde{n}-1)}{4\left(4 \pi^{2}\right)^{3}}\left[\int_{-\infty}^{t_{1}^{-}} d \tau_{2} \int_{t_{1}^{+}}^{t_{2}^{-}} d \tau_{3} Y_{t_{1}^{-} 23}-2 \int_{-\infty}^{t_{1}^{-}} d \tau_{1} \int_{-\infty}^{t_{1}^{-}} d \tau_{2} \int_{t_{1}^{+}}^{t_{2}^{-}} d \tau_{3} \delta\left(\tau_{1}-\tau_{2}\right) Y_{123}\right] \\
& =-\frac{\lambda^{2}(n \cdot \tilde{n}-1)}{4\left(4 \pi^{2}\right)^{3}}\left[\int_{-\infty}^{t_{1}^{-}} d \tau_{2} \int_{t_{1}^{+}}^{t_{2}^{-}} d \tau_{3} Y_{t_{1}^{-} 23}-2 \int_{-\infty}^{t_{1}^{-}} d \tau_{2} \int_{t_{1}^{+}}^{t_{2}^{-}} d \tau_{3} Y_{223}\right] .
\end{aligned}
$$

We thus get

$$
\begin{aligned}
& \mathrm{V} \equiv \mathrm{V} 1+\mathrm{V} 2+\mathrm{V} 3+\mathrm{V} 4+\mathrm{V} 5+\mathrm{V} 6 \\
& =-\frac{\lambda^{2}(n \cdot \tilde{n}-1)}{4\left(4 \pi^{2}\right)^{3}}\left[\int_{-\infty}^{t_{1}^{-}} d \tau_{2} \int_{t_{1}^{+}}^{t_{2}^{-}} d \tau_{3}\left(Y_{t_{1}^{-} 23}+Y_{t_{1}^{+} 23}+Y_{t_{2}^{-} 23}+Y_{t_{2}^{+} 23}\right)\right. \\
& \quad+\int_{t_{1}^{+}}^{t_{2}^{-}} d \tau_{2} \int_{t_{2}^{+}}^{\infty} d \tau_{3}\left(Y_{t_{1}^{-} 23}+Y_{t_{1}^{+} 23}+Y_{t_{2}^{-} 23}+Y_{t_{2}^{+} 23}\right) \\
& \left.\quad-2 \int_{-\infty}^{t_{1}^{-}} d \tau_{2} \int_{t_{1}^{+}}^{t_{2}^{-}} d \tau_{3}\left(Y_{223}+Y_{233}\right)-2 \int_{t_{1}^{+}}^{t_{2}^{-}} d \tau_{2} \int_{t_{2}^{+}}^{\infty} d \tau_{3}\left(Y_{223}+Y_{233}\right)\right] .
\end{aligned}
$$

Note that the last line in this expression contains the function $Y$ evaluated at the coincident points and is therefore divergent. A convenient way to regularize these integrals is to use the dimensional regularization, ${ }^{8}$ which renders $Y$ to be

$$
\begin{aligned}
& Y_{223}^{\epsilon}=\int \frac{d^{4-\epsilon} x_{5}}{x_{25}^{4} x_{35}^{2}}=\frac{\pi^{2-\frac{\epsilon}{2}}}{x_{23}^{2-\epsilon}} \frac{\Gamma\left(1-\frac{\epsilon}{2}\right) \Gamma\left(-\frac{\epsilon}{2}\right) \Gamma\left(1+\frac{\epsilon}{2}\right)}{\Gamma(1-\epsilon)}, \\
& Y_{233}^{\epsilon}=\int \frac{d^{4-\epsilon} x_{5}}{x_{25}^{2} x_{35}^{4}}=Y_{223}^{\epsilon} .
\end{aligned}
$$

\footnotetext{
${ }^{8}$ For derivation of (3.18), see for instance appendix A of [15].
} 

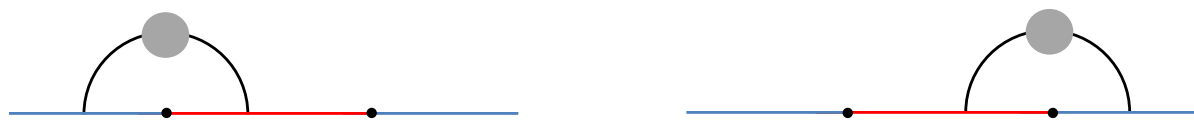

Figure 6. The self-energy diagrams that contribute to the two-loop two-point function. The sum of these two diagrams is given by (3.20).

Self-energy diagrams. We now discuss the contribution from the self-energy diagrams (see figure 6). The one-loop correction to the gauge and the scalar propagators were already computed in the literature and the result in the dimensional regularization reads [14]

$$
\begin{aligned}
& \mu \sim \sim \sim=\frac{g_{\mathrm{YM}}^{4} \delta^{a c} \delta^{b d} \delta_{\mu \nu}}{2\left(4 \pi^{2}\right)^{3}}\left(Y_{223}^{\epsilon}+Y_{233}^{\epsilon}\right), \\
& i \sim j=-\frac{g_{\mathrm{YM}}^{4} \delta^{a c} \delta^{b d} \delta_{i j}}{2\left(4 \pi^{2}\right)^{3}}\left(Y_{223}^{\epsilon}+Y_{233}^{\epsilon}\right) .
\end{aligned}
$$

Here again $\delta^{a c} \delta^{b d}$ is the color factor. ${ }^{9}$ Using these corrected propagators, one can compute the contribution from the self-energy diagrams as follows:

$$
\mathbf{S}=-\frac{\lambda^{2}(n \cdot \tilde{n}-1)}{2\left(4 \pi^{2}\right)^{3}}\left[\int_{-\infty}^{t_{1}^{-}} d \tau_{2} \int_{t_{1}^{+}}^{t_{2}^{-}} d \tau_{3}\left(Y_{223}^{\epsilon}+Y_{233}^{\epsilon}\right)+\int_{t_{1}^{+}}^{t_{2}^{-}} d \tau_{2} \int_{t_{2}^{+}}^{\infty} d \tau_{3}\left(Y_{223}^{\epsilon}+Y_{233}^{\epsilon}\right)\right] .
$$

It is then easy to verify that the contribution from the self-energy diagrams precisely cancels the divergent terms in (3.17).

Using the expression for $Y_{123}$ (3.12), one can straightforwardly evaluate the remaining integral $^{10}$ to get

$$
\mathrm{V}+\mathrm{S}=-2(n \cdot \tilde{n}-1)\left(\frac{\lambda}{8 \pi^{2}}\right)^{2}\left(\frac{\pi^{2}}{3} \log \frac{t_{21}}{\epsilon}+3 \zeta(3)\right)
$$

Final result. Now by summing up all the contributions (3.9) and (3.21), we get the result for the two-point function at two loops:

$$
\begin{gathered}
\left\langle W\left[\mathcal{O}_{1}\left(t_{1}\right) \mathcal{O}_{2}\left(t_{2}\right)\right]\right\rangle_{2 \text {-loop }}=\left(\frac{\lambda}{8 \pi^{2}}\right)^{2}[ \\
(n \cdot \tilde{n}-1)^{2}\left(\frac{\pi^{2}}{6}-2 \log \frac{t_{21}}{\epsilon}+2\left(\log \frac{t_{21}}{\epsilon}\right)^{2}\right) \\
\left.-2(n \cdot \tilde{n}-1)\left(\frac{\pi^{2}}{3} \log \frac{t_{21}}{\epsilon}+3 \zeta(3)\right)\right] .
\end{gathered}
$$

By comparing the weak coupling expansion of the two-point function (2.11), one can finally obtain the two-loop anomalous dimension $\gamma_{j}^{(2)}$ and the constant term $a_{j}^{(2)}$ as follows:

$$
\begin{aligned}
& a^{(2)}=\frac{1}{\left(8 \pi^{2}\right)^{2}}\left[\frac{\pi^{2}}{12}(n \cdot \tilde{n}-1)^{2}-3 \zeta(3)(n \cdot \tilde{n}-1)\right], \\
& \gamma^{(2)}=\frac{1}{\left(8 \pi^{2}\right)^{2}}\left[(n \cdot \tilde{n}-1)^{2}+\frac{\pi^{2}}{3}(n \cdot \tilde{n}-1)\right] .
\end{aligned}
$$

Again, the result for $\gamma^{(2)}$ matches the one in the literature [13].

\footnotetext{
${ }^{9}$ Here we did not write them on the left hand side for simplicity.

${ }^{10}$ In terms of polylogarithms.
} 


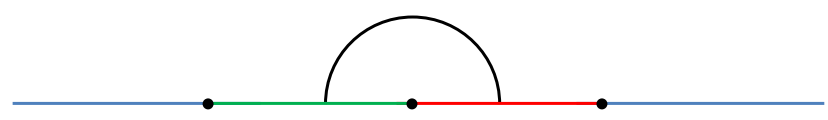

Figure 7. The one-loop diagram for the three-point function which is proportional to $\left(n_{12} \cdot n_{23}-1\right)$. The result of the computation is given in (4.1).

\section{Three-point functions at two loops}

We now set out to compute the three-point functions of DCO's on the Wilson line, given explicitly in (2.2). The strategy of the computation is essentially the same as in the previous section; we list up all possible diagrams and compute each integral explicitly by using appropriate regularisations. Of course, this is easier said than done; the number of diagrams that contribute at a given loop order proliferate as we increase the number of operators.

To circumvent this complication, we use the following trick: when the polarizations of two segments are identical, the quantum correction involving these two segments must vanish owing to the supersymmetry. This implies that the polarization vectors $n_{i j}$ 's enter in the final result only through the combinations ${ }^{11}\left(n_{i j} \cdot n_{k l}-1\right)$. In addition, the final result must be symmetric with respect to the permutation of the operator labels, 1,2 and 3. Therefore, instead of computing all possible diagrams, one can just focus on the coefficients of certain monomials of $\left(n_{i j} \cdot n_{k l}-1\right)$ 's, and symmetrize the resulting expression with respect to the permutation of the operators to get the full answer.

\subsection{One loop}

At one loop, the result is linear in $\left(n_{i j} \cdot n_{k l}-1\right)$. Therefore by using the trick explained above, we can just focus on the term proportional to $\left(n_{12} \cdot n_{23}-1\right)$ and symmetrize the result to get the full expression. As shown in figure 7 , there is only one type of diagrams that produce this contribution. As in the case of the two-point function, one can straightforwardly evaluate them as

$$
\frac{\lambda\left(n_{12} \cdot n_{23}-1\right)}{8 \pi^{2}} \int_{t_{1}^{+}}^{t_{2}^{-}} d \tau_{1} \int_{t_{2}^{+}}^{t_{3}^{-}} d \tau_{2} \frac{1}{\tau_{12}^{2}}=\frac{\lambda\left(n_{12} \cdot n_{23}-1\right)}{8 \pi^{2}} \log \frac{t_{21} t_{32}}{t_{31} \epsilon}
$$

After the symmetrization, we get the full answer,

$$
\left\langle W\left[\mathcal{O}_{1}\left(t_{1}\right) \mathcal{O}_{2}\left(t_{2}\right) \mathcal{O}_{3}\left(t_{3}\right)\right]\right\rangle_{1-\text { loop }}=\frac{\lambda}{8 \pi^{2}} \sum_{\{i, j, k\}}\left(n_{i j} \cdot n_{j k}-1\right) \log \left|\frac{t_{i j} t_{j k}}{t_{k i} \epsilon}\right|,
$$

where the sum is over $\{i, j, k\}=\{1,2,3\},\{2,3,1\},\{3,1,2\}$. Comparing this result with the weak coupling expansion given in (2.11), one can read off the anomalous dimension and the structure constant as ${ }^{12}$

$$
\gamma_{j}^{(1)}=\frac{\left(1-n_{i j} \cdot n_{j k}\right)}{8 \pi^{2}}, \quad c_{123}^{(1)}=0 .
$$

\footnotetext{
${ }^{11}$ At one loop, the result is linear in such combinations while it consists of linear and quadratic pieces at two loops.

${ }^{12}$ Here we already used the fact that the one-loop normalization $a^{(1)}$ vanishes in our scheme. See (3.6).
} 

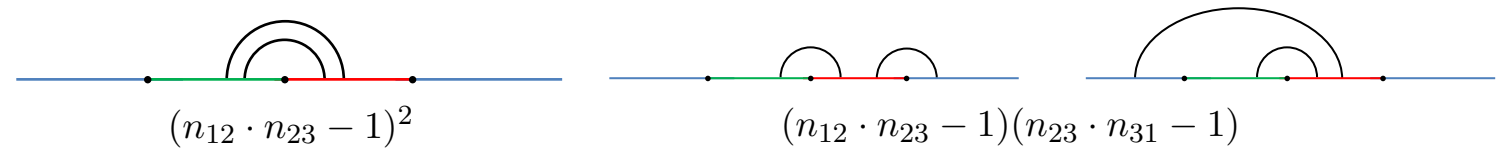

Figure 8. The two-loop ladder diagrams. The left diagram produces the term proportional to $\left(n_{12} \cdot n_{23}-1\right)^{2}$ while the right two diagrams produce the term proportional to $\left(n_{12} \cdot n_{23}-1\right)\left(n_{23}\right.$. $\left.n_{31}-1\right)$.

As expected the result for the anomalous dimension matches the previous result (3.6). This also shows that the one-loop structure constant is exactly zero.

\subsection{Two loops}

At two loops, the result consists of terms quadratic in $\left(n_{i j} \cdot n_{j k}-1\right)$ and terms linear in $\left(n_{i j} \cdot n_{j k}-1\right)$. The quadratic terms come from the ladder-like diagrams while the linear terms arise from the vertex diagrams and the self-energy diagrams.

Let us first compute the quadratic terms. For this purpose, it is enough to compute the terms proportional to $\left(n_{12} \cdot n_{23}-1\right)^{2}$ and $\left(n_{12} \cdot n_{23}-1\right)\left(n_{23} \cdot n_{31}-1\right)$. The diagrams that contribute to these two terms are given in figure 8 . Then, the term proportional to $\left(n_{12} \cdot n_{23}-1\right)^{2}$ can be computed as

$$
\begin{gathered}
\left(\frac{\left(n_{12} \cdot n_{23}-1\right) \lambda}{8 \pi^{2}}\right)^{2} \int_{t_{1}^{+}}^{t_{2}^{-}} d \tau_{1} \int_{\tau_{1}}^{t_{2}^{-}} d \tau_{2} \int_{t_{2}^{+}}^{t_{3}^{-}} d \tau_{3} \int_{\tau_{3}}^{t_{3}^{-}} d \tau_{4} \frac{1}{\tau_{14}^{2}} \frac{1}{\tau_{23}^{2}} \\
=\left(\frac{\left(n_{12} \cdot n_{23}-1\right) \lambda}{8 \pi^{2}}\right)^{2}\left[\frac{\pi^{2}}{6}-\log \left|\frac{t_{12} t_{23}}{t_{31} \epsilon}\right|+\frac{1}{2}\left(\log \left|\frac{t_{12} t_{23}}{t_{31} \epsilon}\right|\right)^{2}\right]
\end{gathered}
$$

On the other hand, the term proportional to $\left(n_{12} \cdot n_{23}-1\right)\left(n_{23} \cdot n_{31}-1\right)$ is given by

$$
\begin{gathered}
\left(n_{12} \cdot n_{23}-1\right)\left(n_{23} \cdot n_{31}-1\right)\left(\frac{\lambda}{8 \pi^{2}}\right)^{2}\left[\int_{t_{1}^{+}}^{t_{2}^{-}} d \tau_{1} \int_{t_{2}^{+}}^{t_{3}^{-}} d \tau_{2} \int_{\tau_{2}}^{t_{3}^{-}} d \tau_{3} \int_{t_{3}^{+}}^{\infty} d \tau_{4} \frac{1}{\tau_{12}^{2}} \frac{1}{\tau_{34}^{2}}\right. \\
\left.+\int_{-\infty}^{t_{1}^{-}} d \tau_{1} \int_{t_{1}^{+}}^{t_{2}^{-}} d \tau_{2} \int_{t_{2}^{+}}^{t_{3}^{+}} d \tau_{3} \int_{\tau_{3}}^{t_{3}^{+}} d \tau_{4} \frac{1}{\tau_{14}^{2}} \frac{1}{\tau_{23}^{2}}\right] \\
=-\left(n_{12} \cdot n_{23}-1\right)\left(n_{23} \cdot n_{31}-1\right)\left(\frac{\lambda}{8 \pi^{2}}\right)^{2}\left(\frac{\pi^{2}}{6}-\log \left|\frac{t_{12} t_{23}}{t_{31} \epsilon}\right| \log \left|\frac{t_{23} t_{31}}{t_{12} \epsilon}\right|\right) .
\end{gathered}
$$

Next we consider the terms linear in $\left(n_{12} \cdot n_{23}-1\right)$. The diagrams that give such contributions are listed in figure 9. The computation of each diagram is a straightforward, yet tedious task. So we relegate the detail of the computation to appendix B and present only the final result here:

$$
-\left(n_{12} \cdot n_{23}-1\right)\left(\frac{\lambda}{8 \pi^{2}}\right)^{2}\left(3 \zeta(3)+\frac{\pi^{2}}{3} \log \left|\frac{t_{12} t_{23}}{t_{31} \epsilon}\right|\right) .
$$




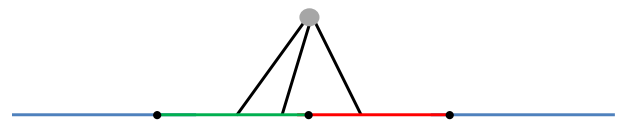

T1

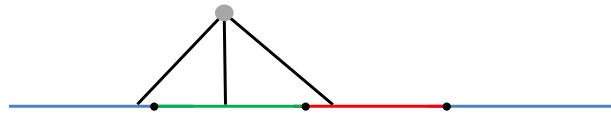

T3

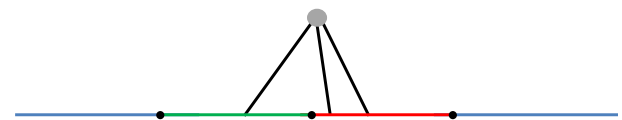

T2

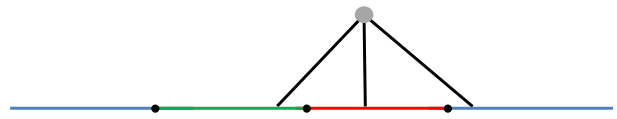

T4

Figure 9. The two-loop diagram with one interaction vertex which produces the term linear in $\left(n_{12} \cdot n_{23}-1\right)$. The computation of each diagram is presented in appendix B.

Then, after the symmetrization, we get

$$
\begin{gathered}
\left\langle W\left[\mathcal{O}_{1}\left(t_{1}\right) \mathcal{O}_{2}\left(t_{2}\right) \mathcal{O}_{3}\left(t_{3}\right)\right]\right\rangle_{2-\operatorname{loop}} \\
=\left(\frac{\lambda}{8 \pi^{2}}\right)^{2} \sum_{\{i, j, k\}}\left[\left(n_{i j} \cdot n_{j k}-1\right)^{2}\left(\frac{\pi^{2}}{6}-\log \left|\frac{t_{i j} t_{j k}}{t_{k i} \epsilon}\right|+\frac{1}{2}\left(\log \left|\frac{t_{i j} t_{j k}}{t_{k i} \epsilon}\right|\right)^{2}\right)\right. \\
\quad-\left(n_{i j} \cdot n_{j k}-1\right)\left(n_{j k} \cdot n_{k i}-1\right)\left(\frac{\pi^{2}}{6}-\log \left|\frac{t_{i j} t_{j k}}{t_{k i} \epsilon}\right| \log \left|\frac{t_{j k} t_{k i}}{t_{i j} \epsilon}\right|\right) \\
\left.-\left(n_{i j} \cdot n_{j k}-1\right)\left(3 \zeta(3)+\frac{\pi^{2}}{3} \log \frac{t_{i j} t_{j k}}{t_{k i} \epsilon}\right)\right] .
\end{gathered}
$$

Here again the sum is over $\{i, j, k\}=\{1,2,3\},\{2,1,3\},\{3,1,2\}$.

Comparing (4.6) with the weak-coupling expansion (2.11), we can read off the two-loop anomalous dimension and the structure constant as follows:

$$
\begin{aligned}
& \gamma_{j}^{(2)}=\frac{1}{\left(8 \pi^{2}\right)^{2}}\left[\left(n_{i j} \cdot n_{j k}-1\right)^{2}+\frac{\pi^{2}}{3}\left(n_{i j} \cdot n_{j k}-1\right)\right], \\
& c_{123}^{(2)}=\frac{1}{\left(8 \pi^{2}\right)^{2}} \frac{\pi^{2}}{12} \sum_{\{i, j, k\}}\left[\left(n_{i j} \cdot n_{j k}-1\right)^{2}-2\left(n_{i j} \cdot n_{j k}-1\right)\left(n_{j k} \cdot n_{k i}-1\right)\right] .
\end{aligned}
$$

As expected, the result for the anomalous dimension matches the one obtained previously in $(3.24)$.

\section{Three-point functions in the ladders limit}

In this section, we consider a special double-scaling limit called the ladders limit. The ladders limit provides an interesting solvable example of defect conformal field theories in higher dimensions: as will be explained more in detail in subsection 5.1, the only diagrams that survive in this limit are the ladder diagrams and one can sum them up by solving the Schwinger-Dyson equation. Such resummation was performed already in the literature to compute the static quark potential and the cusp anomalous dimension [9, 16]. A similar 


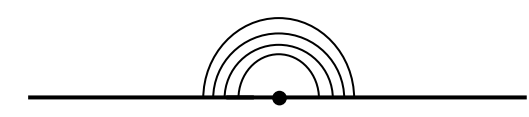

Figure 10. An example of the diagrams that survive in the ladders limit.

technique (or more precisely, a more refined version of it) can be applied to the computation of the structure constant of DCOs as we explain below.

Since this section is rather long, let us give a brief outline of what will be discussed in each subsection. In subsection 5.1, we first explain the set up, namely the ladders limit for the two-point functions and the three-point functions. After doing so, we introduce a building block for the computation, which we call the four-point kernel, in subsection 5.2 and compute it using the Schwinger-Dyson equation. We then compute the two-point function of DCOs and determine their anomalous dimensions and renormalization factors in subsection 5.3. The calculation of the three-point functions are divided into three consecutive subsections 5.4-5.6, depending on the number of DCOs with non-vanishing conformal dimensions.

\section{$5.1 \quad$ Set up}

The ladders limit was introduced in [9] as a double scaling limit in which the 't Hooft coupling constant $\lambda$ goes to zero while the angle between the neighboring polarizations is sent to imaginary negative infinity:

$$
\lambda \rightarrow 0, \quad \hat{\lambda} \equiv \frac{e^{i \theta} \lambda}{4} \sim \frac{\lambda(n \cdot \tilde{n})}{2}: \text { fixed, } \quad(\cos \theta \equiv n \cdot \tilde{n}) .
$$

Since $\lambda$ goes to zero, all the diagrams which contain gluon propagators or interaction vertices disappear in the limit. The only diagrams that survive are the ones which have scalar propagators connecting the two segments since they come with a divergent factor $(n$. $\tilde{n})$ which compensates the vanishing coupling constant. Such diagrams have the structure of the ladders, hence the name. (See figure 10.)

Alternatively, one can define the ladders limit of DCOs directly in the zero-coupling $\mathcal{N}=4 \mathrm{SYM}$ as follows:

$$
\operatorname{Tr}\left[\operatorname{Pexp}\left(\int_{-\infty}^{t} d \tau \hat{\phi}_{i} v^{i}\left|\dot{x}^{\mu}\right|\right) \operatorname{Pexp}\left(\int_{t}^{\infty} d \tau \hat{\phi}_{i} \tilde{v}^{i}\left|\dot{x}^{\mu}\right|\right)\right] .
$$

Here $\hat{\phi}$ is a rescaled scalar field defined by $\hat{\phi} \equiv \phi / \sqrt{\lambda}$ and $v$ and $\tilde{v}$ are complex null vectors related to the effective coupling as $\hat{\lambda}=(v \cdot \tilde{v}) / 2$. The equivalence of the two descriptions can be shown either at the level of diagrams or by carefully taking the limit of the polarization vectors. ${ }^{13}$

For the purpose of computing the (cusp) anomalous dimensions [9], one just needs a two-point function on the Wilson loop, and we only have two polarizations to play with.

\footnotetext{
${ }^{13}$ See the discussion in section 4 of [9].
} 


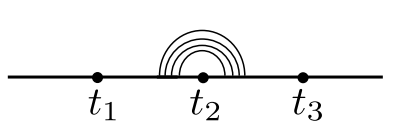

Case I

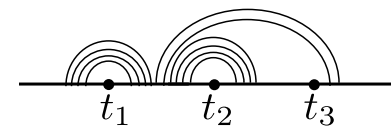

Case II

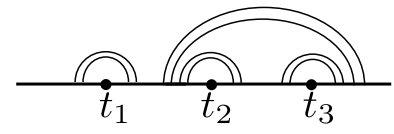

Case III

Figure 11. Examples of diagrams that contribute to the Case I (left), the Case II (middle) and the Case III (right) three-point functions.

On the other hand, the three-point function has three polarizations and we thus have three different angles. We can therefore define three effective couplings ${ }^{14}$

$$
\hat{\lambda}_{i} \equiv \frac{e^{i \theta_{i}} \lambda}{4} \quad(i=1,2,3)
$$

and take various different limits depending on how we scale $\theta_{i}$ 's. The simplest among them is the limit in which one of the angle, say $\theta_{2}$, is sent to infinity while the others are kept finite. In this limit, we have

$$
\text { Case I : } \hat{\lambda}_{1}=\hat{\lambda}_{3}=0, \quad \hat{\lambda}_{2}=\hat{\lambda} \neq 0,
$$

and the diagrams that survive are the ones which connect the two neighboring segments of $\mathcal{O}_{2}$. As we see in subsection 5.3, when the effective coupling is zero, the dimensions of the corresponding DCOs $\left(\mathcal{O}_{1}\right.$ and $\left.\mathcal{O}_{3}\right)$ vanish. In what follows, we call such operators trivial operators. The next simplest limit is the limit,

$$
\text { Case II }: \hat{\lambda}_{1} \neq 0, \quad \hat{\lambda}_{2} \neq 0, \quad \hat{\lambda}_{3}=0 .
$$

We then have two trivial DCOs and one nontrivial DCO and the diagrams are the ones depicted in figure 11. Lastly, there is the most complicated limit, in which all three effective couplings are nonzero.

$$
\text { Case III }: \hat{\lambda}_{1} \neq 0, \quad \hat{\lambda}_{2} \neq 0, \quad \hat{\lambda}_{3} \neq 0 .
$$

These three cases will be discussed separately in subsections 5.4-5.6.

Note that the ladders limit for the three-point function can also be defined directly in the zero-coupling $\mathcal{N}=4 \mathrm{SYM}$. For instance, the Case III corresponds to the correlator,

$$
\begin{aligned}
\left\langle\operatorname { T r } \left[\operatorname{Pexp}\left(\int_{-\infty}^{t_{1}} d \tau \hat{\phi}_{i} v_{31}^{i}\left|\dot{x}^{\mu}\right|\right) \operatorname{Pexp}\left(\int_{t_{1}}^{t_{2}} d \tau \hat{\phi}_{i} v_{12}^{i}\left|\dot{x}^{\mu}\right|\right)\right.\right. \\
\left.\left.\operatorname{Pexp}\left(\int_{t_{2}}^{t_{3}} d \tau \hat{\phi}_{i} v_{23}^{i}\left|\dot{x}^{\mu}\right|\right) \operatorname{Pexp}\left(\int_{t_{3}}^{\infty} d \tau \hat{\phi}_{i} v_{31}^{i}\left|\dot{x}^{\mu}\right|\right)\right]\right\rangle
\end{aligned}
$$

where $v_{i j}$ 's are complex null vectors and related to the effective couplings as $\hat{\lambda}_{j}=\left(v_{i j} \cdot v_{j k}\right) / 2$.

\footnotetext{
${ }^{14}$ See $(2.3)$ for definitions of $\theta_{i}$ 's.
} 

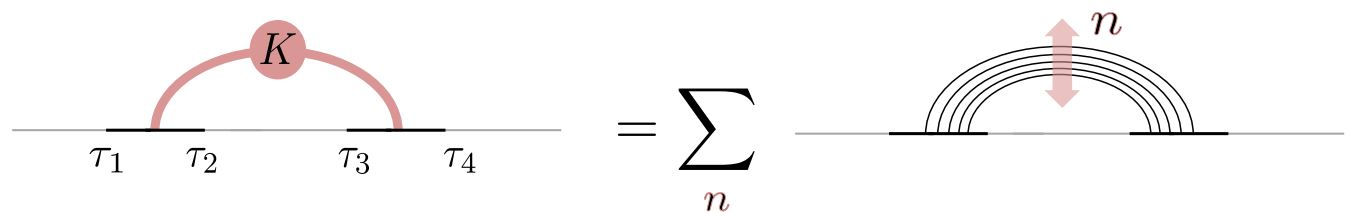

Figure 12. The definition of the four-point kernel. It is defined as a sum over all the ladder diagrams with the endpoints on the black segments in the figure.

\subsection{Four-point kernel and the Schwinger-Dyson equation}

To compute the correlators in the ladders limit, it is useful to introduce a basic building block which we call the four-point kernel $K\left(\tau_{1}, \tau_{2} \mid \tau_{3}, \tau_{4}\right)$. The function $K$ is defined as a sum over all the ladder diagrams with the left endpoints in $\left[\tau_{1}, \tau_{2}\right]$ and the right endpoints in $\left[\tau_{3}, \tau_{4}\right]$. (See also figure 12 )

As shown in figure $13, K\left(\tau_{1}, \tau_{2} \mid \tau_{3}, \tau_{4}\right)$ satisfies the Schwinger-Dyson equation

$$
\begin{aligned}
K\left(\tau_{1}, \tau_{2} \mid \tau_{3}, \tau_{4}\right) & =1+\int_{\tau_{1}}^{\tau_{2}} d s \int_{\tau_{3}}^{\tau_{4}} d t P(t-s) K\left(\tau_{1}, s \mid t, \tau_{4}\right), \\
P(x)\left(=\frac{\lambda}{8 \pi^{2}} \frac{n \cdot \tilde{n}}{x^{2}}\right) & =\frac{\hat{\lambda}}{4 \pi^{2}} \frac{1}{x^{2}},
\end{aligned}
$$

where $P(x)$ is a scalar propagator connecting the two segments, $\left[\tau_{1}, \tau_{2}\right]$ and $\left[\tau_{3}, \tau_{4}\right]$. By differentiating both sides, we can derive a differential equation,

$$
\partial_{\tau_{2}} \partial_{\tau_{3}} K=-P\left(\tau_{3}-\tau_{2}\right) K
$$

One can further simplify this differential equation using the conformal invariance. Owing to the conformal invariance, the kernel $K$ depends on the coordinates only through the cross ratio

$$
u=\frac{\tau_{12} \tau_{34}}{\tau_{13} \tau_{24}}
$$

We can therefore rewrite ${ }^{15}(5.9)$ as a differential equation of one variable, $u$ :

$$
\left[u(1-u) \frac{d^{2}}{d u^{2}}+(1-u) \frac{d}{d u}-\frac{\hat{\lambda}}{4 \pi^{2}(1-u)}\right] K(u)=0 .
$$

Since this is a second-order differential equation, there are two linearly independent solutions. ${ }^{16}$ The correct solution can be selected by imposing the boundary condition, $K(u=0)=1$ (or equivalently $\left.K\right|_{\tau_{1} \rightarrow \tau_{2}}=\left.K\right|_{\tau_{3} \rightarrow \tau_{4}}=1$ ), which comes from the original integral equation (5.8). As a result we have

$$
K(u)=(1-u)_{2}^{-\Omega} F_{1}(-\Omega,-\Omega, 1 ; u),
$$

with

$$
\Omega=\frac{1}{2}\left(-1+\sqrt{1+\frac{\hat{\lambda}}{\pi^{2}}}\right)
$$




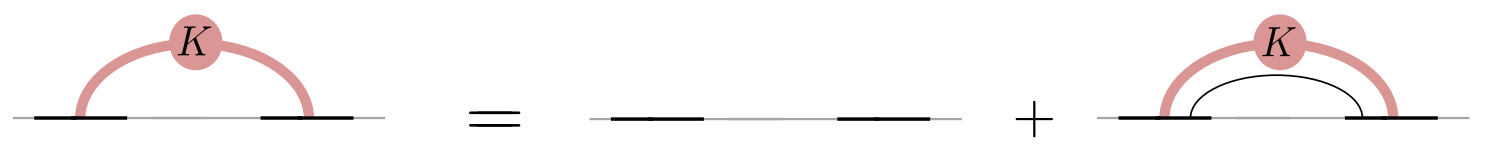

Figure 13. The Schwinger-Dyson equation satisfied by the four-point kernel $K$.
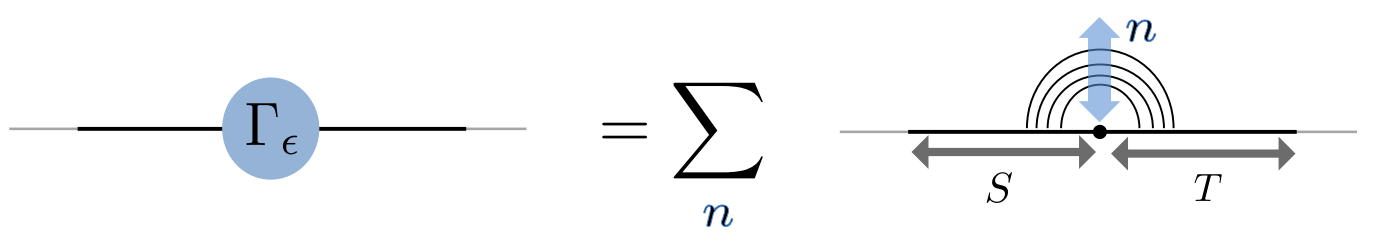

Figure 14. The definition of the (regularized) vertex function. It is given by a sum of diagrams whose end-points are in $[-S,-\epsilon / 2]$ and $[\epsilon / 2, T]$

Using the four-point kernel, one can compute other physically important quantities. One such quantity is the vertex function $\Gamma_{\epsilon}(S, T)$, depicted in figure 14. Roughly speaking, it is given by a sum over the ladder diagrams whose end points are in $[-S, 0]$ and $[0, T]$. This quantity is however UV divergent and one has to introduce a point-splitting cut off to regularize such divergence. Thus, the precise definition is given by

$$
\Gamma_{\epsilon}(S, T) \equiv K(-S,-\epsilon / 2 \mid \epsilon / 2, T)=K\left(\frac{\left(S-\frac{\epsilon}{2}\right)\left(T-\frac{\epsilon}{2}\right)}{\left(S+\frac{\epsilon}{2}\right)\left(T+\frac{\epsilon}{2}\right)}\right)
$$

Here the subscript $\epsilon$ is introduced to remember that it is a "bare" quantity which depends explicitly on the cut off. Note that the vertex function also satisfies the differential equation

$$
\partial_{S} \partial_{T} \Gamma_{\epsilon}(S, T)=P(S+T) \Gamma_{\epsilon}(S, T),
$$

From the explicit form of the four-point kernel, one can determine the leading behavior of $\Gamma_{\epsilon}$ in the limit $S, T \gg \epsilon$,

$$
\Gamma_{\epsilon}(S, T)=\underbrace{\frac{A(\Omega)}{\epsilon^{\Omega}\left(\frac{1}{S}+\frac{1}{T}\right)^{-\Omega}}}_{\equiv \Gamma_{\mathrm{IR}}(S, T)}+O(\epsilon), \quad A(\Omega)=\frac{\Gamma(2 \Omega+1)}{\Gamma(\Omega+1)^{2}} .
$$

As discussed in appendix $\mathrm{C}$, there is an intriguing relation between the vertex function and the solutions to the Schrödinger equation (also called the Bethe-Salpeter equation) studied in $[9,16]$ : by rewriting it in a different coordinate, one can explicitly show that the leading term $\Gamma_{\mathrm{IR}}$ is related to the ground-state wave function of the Schrödinger equation. Furthermore, it turns out that the subleading terms in the expansion correspond to the excited-state wave functions of the same Schrödinger equation. In this sense, the full vertex function $\Gamma_{\epsilon}$ can be regarded as a generating function of such wave functions. From the defect CFT point of view, the expansion of $\Gamma_{\epsilon}$ in $\epsilon$ is nothing but the OPE expansion of the

\footnotetext{
${ }^{15}$ To rewrite the differential equation, we used $\partial_{\tau_{2}}=-\tau_{14} /\left(\tau_{12} \tau_{24}\right) \partial_{u}$ and $\partial_{\tau_{3}}=\tau_{14} /\left(\tau_{13} \tau_{34}\right) \partial_{u}$

${ }^{16}$ The other (incorrect) solution is $(1-u)^{-\Omega}\left[{ }_{2} F_{1}^{*}(-\Omega,-\Omega, 1 ; u)+\log u{ }_{2} F_{1}(-\Omega,-\Omega, 1 ; u)\right]$, with ${ }_{2} F_{1}^{*}(a, b, c ; u)=\left(\partial_{a}+\partial_{b}+2 \partial_{c}\right)_{2} F_{1}^{*}(a, b, c ; u)$.
} 


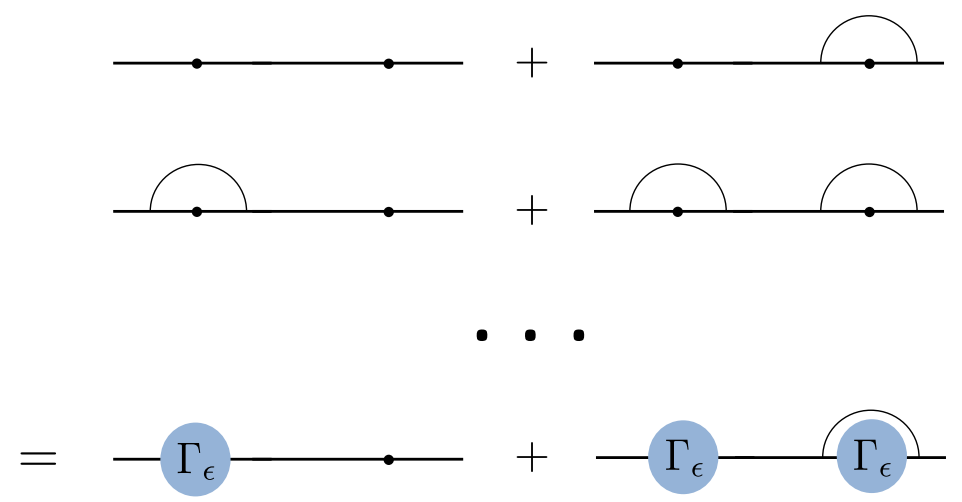

Figure 15. The resummation of the ladder diagrams for the two-point function. The contribution from the left figure on the last line will be denoted by 1st while the one from the right figure will be denoted as 2nd.

"four-point function" $K$, with its leading term controlled by a conformal primary DCO and the subleading terms controlled by its descendants. This provides a physical interpretation of the solutions to the Schrödinger equation: namely the ground state corresponds to a conformal primary and the excited states correspond to its descendants. For a more detailed account on this point, see appendix C.

\subsection{Two-point functions and renormalization}

As in the two-loop analysis performed in the preceding sections, to extract the scheme independent structure constant, one first needs to determine the renormalization factor $Z^{-1 / 2}$ :

$$
\mathcal{O}=Z^{-1 / 2} \mathcal{O}^{B}
$$

Here $\mathcal{O}^{B}$ denotes the bare DCO while $\mathcal{O}$ denotes the renormalized DCO. The renormalization factor is determined by requiring that the renormalized two-point function has a canonical form:

$$
\left\langle\mathcal{O}\left(\tau_{1}\right) \mathcal{O}\left(\tau_{2}\right)\right\rangle=Z^{-1}\left\langle\mathcal{O}^{B}\left(\tau_{1}\right) \mathcal{O}^{B}\left(\tau_{2}\right)\right\rangle=\frac{1}{\left|\tau_{12}\right|^{2 \Delta}}
$$

In what follows, we evaluate the two-point function of the bare operators and determine $Z$.

The two-point function can be computed using the vertex functions as shown in figure 15. The contribution from each diagram is given as follows:

$$
\begin{aligned}
& \text { 1st }=\Gamma_{\epsilon}\left(\infty, \tau_{21}\right), \\
& \text { 2nd }=\int_{\tau_{1}^{+}}^{\tau_{2}^{-}} d s \int_{\tau_{2}^{+}}^{\infty} d t \Gamma_{\epsilon}\left(\infty, s-\tau_{1}\right) P(t-s) \Gamma_{\epsilon}\left(\tau_{2}-s, t-\tau_{2}\right) .
\end{aligned}
$$

As in the preceding sections, the endpoints are regularized as $\tau_{i}^{ \pm}=\tau_{i} \pm \epsilon / 2$. The first contribution can be approximated by $\Gamma_{\mathrm{IR}}$ and is given by

$$
\text { 1st } \sim\left(\frac{\tau_{21}}{\epsilon}\right)^{\Omega} \text {. }
$$


To evaluate the second contribution, we use the differential equation (5.15) and rewrite it as

$$
\begin{aligned}
2 \mathrm{nd} & =\int_{\tau_{1}^{+}}^{\tau_{2}^{-}} d s \int_{\tau_{2}^{+}}^{\infty} d t \Gamma_{\epsilon}\left(\infty, s-\tau_{1}\right)\left(-\partial_{s} \partial_{t}\right) \Gamma_{\epsilon}\left(\tau_{2}-s, t-\tau_{2}\right) \\
& =-\int_{\tau_{1}^{+}}^{\tau_{2}^{-}} d s \Gamma_{\epsilon}\left(\infty, s-\tau_{1}\right) \partial_{s} \Gamma_{\epsilon}\left(\tau_{2}-s, \infty\right)
\end{aligned}
$$

In passing to the second line, we used $\Gamma_{\epsilon}(*, \epsilon / 2)=\left.K(u)\right|_{u=0}=0$. Unlike the first contribution, there is a priori no reason to expect that $\Gamma_{\epsilon}$ can be approximated by $\Gamma_{\mathrm{IR}}$ in (5.21) since the arguments of $\Gamma_{\epsilon}$ can be of order $O(\epsilon)$. Nevertheless, it turns out that the leading singular piece in the limit $\epsilon \ll 1$ can be computed by replacing $\Gamma_{\epsilon}$ with $\Gamma_{\text {IR }}$. Roughly speaking, this is because the difference between $\Gamma_{\epsilon}$ and $\Gamma_{\text {IR }}$,

$$
\Gamma_{\mathrm{UV}} \equiv \Gamma_{\epsilon}-\Gamma_{\mathrm{IR}}
$$

is of order $\epsilon \Gamma_{\mathrm{IR}}$ whenever the arguments are $O(1)$ while it is of $O(1)$ only when the arguments are in a small interval of length $\epsilon$ near the origin. Therefore the contribution from $\Gamma_{\mathrm{UV}}$ is always $O(\epsilon)$ smaller than the contribution from $\Gamma_{\mathrm{IR}}$. See appendix D for more detailed arguments.

Once we replace $\Gamma_{\epsilon}$ with $\Gamma_{\mathrm{IR}}$, the computation is straightforward:

$$
\text { 2nd } \begin{aligned}
& \stackrel{\leftrightarrow}{=}-\left(\frac{A(\Omega)}{\epsilon^{\Omega}}\right)^{2} \int_{\tau_{1}}^{\tau_{2}} d s\left(s-\tau_{1}\right)^{\Omega} \partial_{s}\left(\tau_{2}-s\right)^{\Omega}=\left(\frac{A(\Omega)}{\epsilon^{\Omega}}\right)^{2} \Omega \int_{0}^{1} d \bar{s} \bar{s}^{\Omega}(1-\bar{s})^{\Omega-1} \\
& =\frac{\Gamma(2 \Omega+1)}{\Gamma(\Omega+1)^{2}} \frac{\left|\tau_{12}\right|^{2 \Omega}}{\epsilon^{2 \Omega}} .
\end{aligned}
$$

In the second equality, we performed the transformation $\bar{s}=\left(s-\tau_{1}\right) /\left(\tau_{1}-\tau_{2}\right)$ and in the last equality we used

$$
I(a, b) \equiv b \int_{0}^{1} d \bar{s} \bar{s}^{a}(1-\bar{s})^{b-1}=\frac{\Gamma(a+1) \Gamma(b+1)}{\Gamma(a+b+1)} .
$$

We thus obtain

$$
\left\langle\mathcal{O}^{B}\left(\tau_{1}\right) \mathcal{O}^{B}\left(\tau_{2}\right)\right\rangle \stackrel{\epsilon \lll 1}{=} \frac{\Gamma(2 \Omega+1)}{\Gamma(\Omega+1)^{2}} \frac{\left|\tau_{12}\right|^{2 \Omega}}{\epsilon^{2 \Omega}} .
$$

Note that the first contribution 1st only gives a subleading correction. By comparing (5.26) with (5.18), we conclude that the conformal dimension and the renormalization factor of the DCO are given by ${ }^{17}$

$$
\Delta(\hat{\lambda})=-\Omega, \quad Z(\hat{\lambda})=\frac{A(\Omega)}{\epsilon^{2 \Omega}} .
$$

As expected, the result for the conformal dimension matches the one in [9].

Before ending this subsection, let us make a further comment on $\Gamma_{\epsilon}$ and $\Gamma_{\mathrm{IR}}$. As explained above, the leading term in $\epsilon \ll 1$ limit can be computed by replacing $\Gamma_{\epsilon}$ 's

\footnotetext{
${ }^{17}$ Note that the conformal dimension of the DCO is negative. This however is not a contradiction since the defect CFT we are studying lacks unitarity.
} 


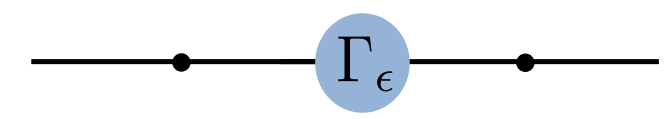

Figure 16. The resummation of the ladder diagrams for the Case I three-point function.

with $\Gamma_{\text {IR }}$ 's in the diagram with the maximal number of vertex functions. As discussed in appendix $\mathrm{D}$, this is rather an universal phenomenon and it applies also to the computation of the multi-point functions. When the effect of the renormalization is taken into account, this leads to the following recipe of computing the renormalized correlation functions:

1. List up all the diagrams that compute the bare correlators and select those with a maximal number of vertex functions.

2. Replace $\Gamma_{\epsilon}$ in those diagrams with the renormalized vertex function $\Gamma^{R}$

$$
\Gamma^{R}(S, T) \equiv \lim _{\epsilon \rightarrow 0} Z^{-1 / 2} \Gamma_{\mathrm{IR}}(S, T)=\sqrt{A(\Omega)}\left(\frac{1}{S}+\frac{1}{T}\right)^{-\Omega}
$$

and compute the integrals.

In the subsections below, we compute the structure constants following this recipe.

\subsection{Case I: 1 nontrivial and 2 trivial DCOs}

We now compute the three-point function of one nontrivial DCO and two trivial DCOs. For simplicity we first consider the case where the operator in the middle is a nontrivial DCO (see figure 16). In this case, one can easily resum the diagrams by a single vertex function. Thus, following the prescription at the end of the previous subsection, we obtain

$$
\begin{aligned}
\left\langle\mathcal{O}_{1}^{\circ}\left(\tau_{1}\right) \mathcal{O}_{2}^{\bullet}\left(\tau_{2}\right) \mathcal{O}_{3}^{\circ}\left(\tau_{3}\right)\right\rangle & =\Gamma^{R}\left(\tau_{21}, \tau_{32}\right)=\frac{C_{\circ \bullet}}{\tau_{21}^{-\Omega} \tau_{32}^{-\Omega} \tau_{31}^{\Omega}}, \\
C_{\circ \bullet \bullet} & =\sqrt{A(\Omega)}=\frac{\Gamma(2 \Omega+1)^{1 / 2}}{\Gamma(\Omega+1)} .
\end{aligned}
$$

Here and in what follows, the symbols $\circ$ and $\bullet$ signify a trivial DCO and a nontrivial DCO respectively. At weak coupling, the result can be expanded from (5.13) as

$$
C_{\circ \bullet \circ}=1+\frac{\pi^{2}}{12}\left(\frac{\hat{\lambda}}{4 \pi^{2}}\right)^{2}-\left(\frac{\pi^{2}}{6}+\zeta(3)\right)\left(\frac{\hat{\lambda}}{4 \pi^{2}}\right)^{3}+O\left(\hat{\lambda}^{4}\right) .
$$

The result up to two loops reproduces the perturbative result for the ladder diagrams in the previous section. At strong coupling $(\hat{\lambda} \gg 1)$, on the other hand, the result exponentiates and is given by

$$
\ln C_{\circ \bullet \circ} \sim \frac{\sqrt{\hat{\lambda}}}{2 \pi} \ln 2 .
$$

The structure of the result suggests the existence of some dual description in terms of classical string with the tension $\sqrt{\hat{\lambda}}$. It would be interesting to look for such a description which reproduces this result. 

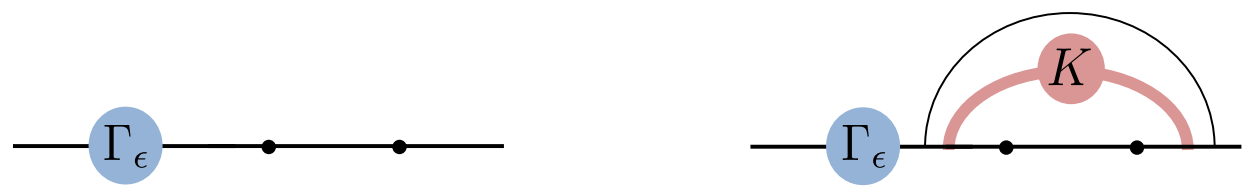

Figure 17. The resummation of the ladder diagrams for the Case I three-point function when the leftmost operator is the nontrivial DCO. Unlike the one in figure 16, the result is given by a sum of two diagrams shown above (although the structure constant itself must be the same).

As a consistency check, let us also compute the three-point function in which the rightmost operator is a nontrivial DCO $\left(C_{\bullet \bullet \circ}\right)$. Although the result must be equal to $C_{\circ \bullet}$ owing to the permutation symmetry, the diagrams are not identical as shown in figure 17 . Written explicitly, we have

$$
\frac{C \bullet \circ}{\tau_{21}^{-\Omega} \tau_{32}^{\Omega} \tau_{31}^{-\Omega}}=\Gamma^{R}\left(\infty, \tau_{21}\right)+\underbrace{\frac{\hat{\lambda}}{4 \pi^{2}} \int_{\tau_{1}}^{\tau_{2}} d s \int_{\tau_{3}}^{\infty} d t \frac{\Gamma^{R}\left(\infty, s-\tau_{1}\right) K\left(s, \tau_{2} \mid \tau_{3}, t\right)}{(t-s)^{2}}}_{(*)} .
$$

Using the Schwinger-Dyson equation (5.9), one can rewrite the second term on the right hand side as

$$
(*)=-\int_{\tau_{1}}^{\tau_{2}} d s \int_{\tau_{3}}^{\infty} d t \Gamma^{R}\left(\infty, s-\tau_{1}\right) \partial_{s} \partial_{t} K\left(s, \tau_{2} \mid \tau_{3}, t\right) .
$$

Clearly, the $t$ integral can be trivially performed and we get

$$
(*)=\int_{\tau_{1}}^{\tau_{2}} d s \partial_{s}\left(1-K\left(s, \tau_{2} \mid \tau_{3}, \infty\right)\right) \Gamma^{R}\left(\infty, s-\tau_{1}\right),
$$

where we used $K\left(*, *^{\prime} \mid x, x\right)=1$. By performing the integration by parts and adding the first term in (5.32), we arrive at a simple formula

$$
\frac{C_{\bullet \circ}}{\tau_{21}^{-\Omega} \tau_{32}^{\Omega} \tau_{31}^{-\Omega}}=\int_{\tau_{1}}^{\tau_{2}} d s \partial_{s} \Gamma^{R}\left(\infty, s-\tau_{1}\right) K\left(s, \tau_{2} \mid \tau_{3}, \infty\right) .
$$

As shown in appendix E, this integral can be evaluated explicitly using the identities of the hypergeometric functions and we obtain the expected result

$$
C_{\bullet \circ \circ}=C_{\circ \bullet \circ}=\sqrt{A(\Omega)} .
$$

\subsection{Case II: 2 nontrivial and 1 trivial DCOs}

Let us now compute the structure constant of the case II correlators,

$$
\left\langle\mathcal{O}_{1}^{\bullet}\left(\tau_{1}\right) \mathcal{O}_{2}^{\bullet}\left(\tau_{2}\right) \mathcal{O}_{3}^{\circ}\left(\tau_{3}\right)\right\rangle=\frac{C_{\bullet \bullet \circ}}{\tau_{21}^{-\Omega_{1}-\Omega_{2}} \tau_{32}^{\Omega_{1}-\Omega_{2}} \tau_{31}^{-\Omega_{1}+\Omega_{2}}} .
$$

Since we have two effective couplings $\hat{\lambda}_{1,2}$ in this case, we shall distinguish various functions of the effective couplings by putting subscripts; for instance the dimension of each operator will be denoted as $\Delta_{i}=-\Omega_{i} \equiv-\Omega\left(\hat{\lambda}_{i}\right)$. 

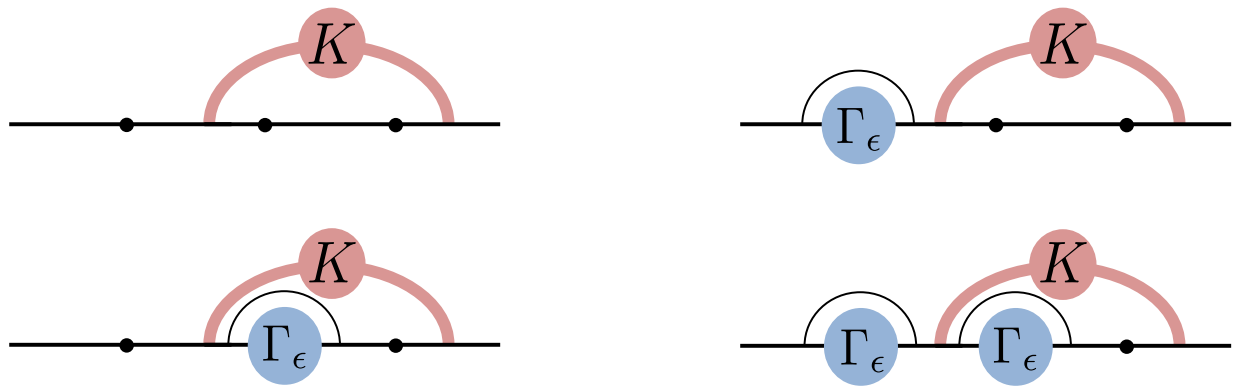

Figure 18. The resummation of the ladder diagrams for the Case II three-point function. Among the four diagrams, only the last one gives a dominant contribution in the limit $\epsilon \rightarrow 0$.

The diagrams that contribute to the bare three-point functions are listed in figure 18 . Following the recipe in subsection 5.3, we just consider the diagram with a maximal number of vertex functions, which in this case is the last diagram. We then get the following expression for the renormalized three-point function ${ }^{18}$

$$
\begin{aligned}
\left\langle\mathcal{O}_{1}^{\bullet} \mathcal{O}_{2}^{\bullet} \mathcal{O}_{3}^{\circ}\right\rangle=\int_{\tau_{1}}^{\tau_{2}} d s \int_{\tau_{2}}^{\tau_{3}} d t \int_{-\infty}^{\tau_{1}} d u \int_{\tau_{1}}^{s} d v & \left(-\partial_{u} \partial_{v}\right) \Gamma_{1}^{R}\left(\tau_{1}-u, v-\tau_{1}\right) \\
& \times K_{1}\left(v, s \mid \tau_{3}, \infty\right)\left(-\partial_{s} \partial_{t}\right) \Gamma_{2}^{R}\left(\tau_{2}-s, t-\tau_{2}\right) .
\end{aligned}
$$

Here we have already replaced the scalar propagators with the derivatives using the equation (5.9). In (5.38), the integral of $u$ can be performed straightforwardly. After doing so, the integral of $v$ becomes

$$
\int_{\tau_{1}}^{s} d v \partial_{v} \Gamma_{1}^{R}\left(\infty, v-\tau_{1}\right) K_{1}\left(v, s \mid \tau_{3}, \infty\right)
$$

This integral is essentially the same as the right hand side of (5.35). Therefore, using the result there, we can evaluate it to get

$$
\begin{aligned}
\left\langle\mathcal{O}_{1}^{\bullet} \mathcal{O}_{2}^{\bullet} \mathcal{O}_{3}^{\circ}\right\rangle & =\tau_{31}^{\Omega_{1}} \sqrt{A\left(\Omega_{1}\right)} \int_{\tau_{1}}^{\tau_{2}} d s \int_{\tau_{2}}^{\tau_{3}} d t\left(\frac{s-\tau_{1}}{\tau_{3}-s}\right)^{\Omega_{1}}\left(-\partial_{s} \partial_{t}\right) \Gamma_{2}^{R}\left(\tau_{2}-s, t-\tau_{2}\right) \\
& =\tau_{31}^{\Omega_{1}} \sqrt{A\left(\Omega_{1}\right) A\left(\Omega_{2}\right)} \int_{\tau_{1}}^{\tau_{2}} d s \int_{\tau_{2}}^{\tau_{3}} d t\left(\frac{s-\tau_{1}}{\tau_{3}-s}\right)^{\Omega_{1}}\left(-\partial_{s} \partial_{t}\right)\left(\frac{\left(\tau_{2}-s\right)\left(t-\tau_{2}\right)}{t-s}\right)^{\Omega_{2}} .
\end{aligned}
$$

We can now perform the $t$ integral to get

$$
\left\langle\mathcal{O}_{1}^{\bullet} \mathcal{O}_{2}^{\bullet} \mathcal{O}_{3}^{\circ}\right\rangle=-\tau_{31}^{\Omega_{1}} \tau_{32}^{\Omega_{2}} \sqrt{A\left(\Omega_{1}\right) A\left(\Omega_{2}\right)} \int_{\tau_{1}}^{\tau_{2}} d s\left(\frac{s-\tau_{1}}{\tau_{3}-s}\right)^{\Omega_{1}} \partial_{s}\left(\frac{\tau_{2}-s}{\tau_{3}-s}\right)^{\Omega_{2}}
$$

The last integral can be done explicitly ${ }^{19}$ by performing the following change of variables, which amounts to performing the Möbius transformation $\left(\tau_{1}, \tau_{2}, \tau_{3}\right) \rightarrow(0,1, \infty)$ :

$$
\bar{s}=\frac{s-\tau_{1}}{s-\tau_{3}} \frac{\tau_{2}-\tau_{3}}{\tau_{2}-\tau_{1}} .
$$

\footnotetext{
${ }^{18}$ As mentioned above, the notations $\Gamma_{i}^{R}$ and $K_{i}$ mean $\Gamma^{R}\left(\hat{\lambda}_{i}\right)$ and $K\left(\hat{\lambda}_{i}\right)$.

${ }^{19}$ The integral reduces to the integral for $I(a, b)$ given in $(5.25)$.
} 
As a result, we get

$$
C_{\bullet \bullet \circ}=\sqrt{A\left(\Omega_{1}\right) A\left(\Omega_{2}\right)} I\left(\Omega_{1}, \Omega_{2}\right)=\frac{\Gamma\left(2 \Omega_{1}+1\right)^{1 / 2} \Gamma\left(2 \Omega_{2}+1\right)^{1 / 2}}{\Gamma\left(\Omega_{1}+\Omega_{2}+1\right)} .
$$

Note that the result (correctly) reduces to the one in the previous subsection if we set $\Omega_{2}=0$. At weak coupling, the result reads

$$
C \bullet \bullet \circ=1+\frac{\left(\hat{\lambda}_{1}-\hat{\lambda}_{2}\right)^{2}}{192 \pi^{2}}+\frac{\left(\hat{\lambda}_{1}-\hat{\lambda}_{2}\right)^{2}\left(\hat{\lambda}_{1}+\hat{\lambda}_{2}\right)\left(\pi^{2}+6 \zeta(3)\right)}{384 \pi^{6}}+O\left(\hat{\lambda}^{4}\right),
$$

whereas at strong coupling it reads

$$
\ln C \bullet \bullet \sim \Omega_{1} \ln \frac{2 \Omega_{1}}{\Omega_{1}+\Omega_{2}}+\Omega_{2} \ln \frac{2 \Omega_{2}}{\Omega_{1}+\Omega_{2}}, \quad \Omega_{i} \sim \frac{\sqrt{\hat{\lambda}_{i}}}{2 \pi} .
$$

The result at two loops at weak coupling matches the ladder contribution to the perturbative result given in section 4 .

\subsection{Case III: 3 nontrivial DCOs}

In this subsection, we compute the most general three-point functions of DCOs,

$$
\left\langle\mathcal{O}_{1}^{\bullet}\left(\tau_{1}\right) \mathcal{O}_{2}^{\bullet}\left(\tau_{2}\right) \mathcal{O}_{3}^{\bullet}\left(\tau_{3}\right)\right\rangle=\frac{C_{\bullet \bullet \bullet}}{\tau_{21}^{-\Omega_{1}-\Omega_{2}+\Omega_{3}} \tau_{32}^{-\Omega_{2}-\Omega_{3}+\Omega_{1}} \tau_{31}^{-\Omega_{3}-\Omega_{1}+\Omega_{2}}} .
$$

As shown in figure 19, there are several different diagrams that contribute to this threepoint function. However, only three diagrams ( $\mathrm{a}, \mathrm{b}$ and $\mathrm{c}$ in figure 19) contain a maximal number of vertex functions. Below we compute the contributions from these diagrams following the recipe in subsection 5.3.

The contribution from the diagram a is given by

$$
\begin{aligned}
\mathrm{a}=(-1) \times \int_{\tau_{1}}^{\tau_{2}} d s \int_{\tau_{2}}^{\tau_{3}} d t \int_{t}^{\tau_{3}} d u \int_{\tau_{3}}^{\infty} d v \Gamma^{R}(\infty, s- & \left.\tau_{1}\right)\left(-\partial_{s} \partial_{t}\right) \Gamma^{R}\left(\tau_{2}-s, t-\tau_{2}\right) \\
& \times\left(-\partial_{u} \partial_{v}\right) \Gamma^{R}\left(\tau_{3}-u, v-\tau_{3}\right) .
\end{aligned}
$$

Here again we have already replaced the scalar propagators with the derivatives using (5.9). Since the integrand is a total derivative with respect to $u$ and $v$, one can perform those integrals to get

$$
\begin{aligned}
& \mathrm{a}=-I_{1} \\
& I_{1} \equiv \prod_{i=1}^{3} \sqrt{A\left(\Omega_{i}\right)} \int_{\tau_{1}}^{\tau_{2}} d s \int_{\tau_{2}}^{\tau_{3}} d t(s-t)^{\Omega_{1}}\left(-\partial_{s} \partial_{t}\right)\left(\frac{\left(\tau_{2}-s\right)\left(t-\tau_{2}\right)}{t-s}\right)^{\Omega_{2}}\left(\tau_{3}-t\right)^{\Omega_{3}} .
\end{aligned}
$$

Next we consider the contribution from the diagram $b$, which is given by

$$
\begin{aligned}
\mathrm{b}= & \int_{\tau_{1}}^{\tau_{2}} d s \int_{\tau_{2}}^{\tau_{3}} d t \int_{\tau_{3}}^{\infty} d u \int_{t}^{\tau_{3}} d v \int_{-\infty}^{\tau_{1}} d w \int_{\tau_{1}}^{s} d x K(x, s \mid u, \infty) \\
& \times\left(-\partial_{w} \partial_{x}\right) \Gamma^{R}\left(w-\tau_{1}, x-\tau_{1}\right)\left(-\partial_{s} \partial_{t}\right) \Gamma^{R}\left(\tau_{2}-s, t-\tau_{2}\right)\left(-\partial_{u} \partial_{v}\right) \Gamma^{R}\left(\tau_{3}-v, u-\tau_{3}\right) .
\end{aligned}
$$




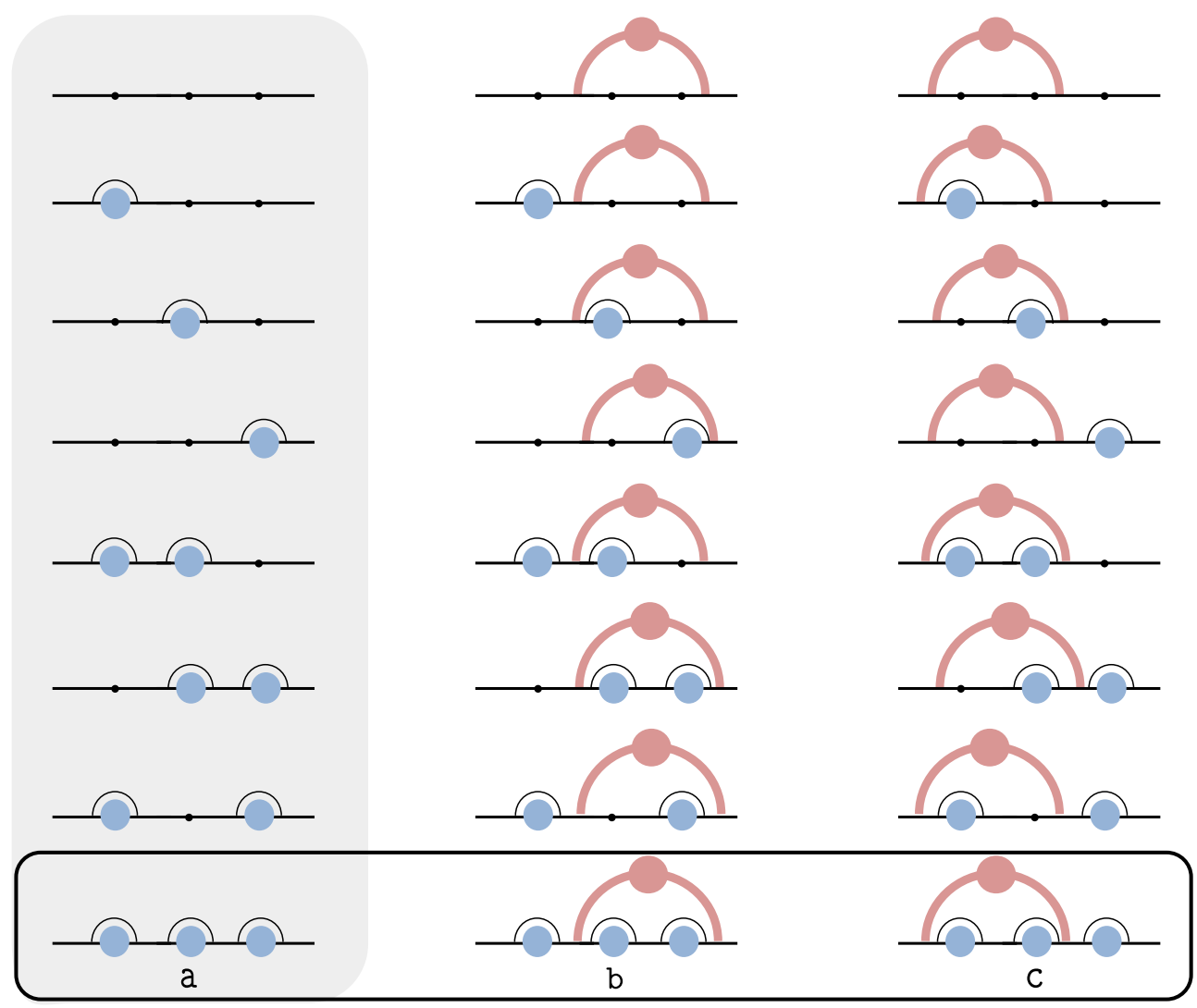

Figure 19. The resummation of the ladder diagrams for the Case III three-point function. To avoid the double-counting, the diagrams in the first column (depicted in the gray background) must be summed with a negative sign. Among these diagrams only the last three (encircled by the thick black line) contribute dominantly in the limit $\epsilon \rightarrow 0$.

By performing the trivial integrals of $v$ and $w$, we get the integrand with $\partial_{u} \Gamma^{R}\left(\infty, u-\tau_{3}\right)$ and $\partial_{x} \Gamma^{R}\left(\infty, x-\tau_{1}\right)$. Then, the integral of $x$ coincides with the one we already studied in (5.35). We can thus evaluate it to obtain

$$
\begin{aligned}
& \mathrm{b}=\prod_{i=1}^{3} \sqrt{A\left(\Omega_{i}\right)} \int_{\tau_{1}}^{\tau_{2}} d s \int_{\tau_{2}}^{\tau_{3}} d t \int_{\tau_{3}}^{\infty} d u\left(\frac{\left(s-\tau_{1}\right)\left(u-\tau_{1}\right)}{u-s}\right)^{\Omega_{1}} \\
& \times\left(-\partial_{s} \partial_{t}\right)\left(\frac{\left(\tau_{2}-s\right)\left(t-\tau_{2}\right)}{t-s}\right)^{\Omega_{2}} \partial_{u}\left(\frac{\left(\tau_{3}-t\right)\left(u-\tau_{3}\right)}{u-t}\right)^{\Omega_{3}} .
\end{aligned}
$$

After doing so, we perform integration by parts for the $u$ integral. We then get $I_{1}$ as a surface term and the full result reads

$$
\mathrm{b}=I_{1}+I_{2},
$$

with

$$
\begin{aligned}
I_{2}=\prod_{i=1}^{3} \sqrt{A\left(\Omega_{i}\right)} \int_{\tau_{1}}^{\tau_{2}} d s \int_{\tau_{2}}^{\tau_{3}} d t \int_{\tau_{3}}^{\infty} d u\left(-\partial_{u}\right)\left(\frac{\left(s-\tau_{1}\right)\left(u-\tau_{1}\right)}{u-s}\right)^{\Omega_{1}} \\
\\
\quad \times\left(-\partial_{s} \partial_{t}\right)\left(\frac{\left(\tau_{2}-s\right)\left(t-\tau_{2}\right)}{t-s}\right)^{\Omega_{2}}\left(\frac{\left(\tau_{3}-t\right)\left(u-\tau_{3}\right)}{u-t}\right)^{\Omega_{3}} .
\end{aligned}
$$


The contribution from the diagram c can be evaluated in a similar manner and the result reads

$$
\begin{aligned}
\mathbf{c}=I_{3} \equiv \prod_{i=1}^{3} \sqrt{A\left(\Omega_{i}\right)} \int_{\tau_{1}}^{\tau_{2}} d s \int_{\tau_{2}}^{\tau_{3}} d t \int_{-\infty}^{\tau_{1}} d u\left(-\partial_{u}\right)\left(\frac{\left(s-\tau_{1}\right)\left(u-\tau_{1}\right)}{u-s}\right)^{\Omega_{1}} \\
\\
\quad \times\left(-\partial_{s} \partial_{t}\right)\left(\frac{\left(\tau_{2}-s\right)\left(t-\tau_{2}\right)}{t-s}\right)^{\Omega_{2}}\left(\frac{\left(\tau_{3}-t\right)\left(u-\tau_{3}\right)}{u-t}\right)^{\Omega_{3}} .
\end{aligned}
$$

Summing up three contributions, we get

$$
\left\langle\mathcal{O}_{1}^{\bullet} \mathcal{O}_{2}^{\bullet} \mathcal{O}_{3}^{\bullet}\right\rangle=\mathrm{a}+\mathrm{b}+\mathrm{c}=I_{2}+I_{3}
$$

To further proceed, we perform the following change of variables,

$$
\bar{s}=\frac{\tau_{23}}{\tau_{21}} \frac{s-\tau_{1}}{s-\tau_{3}}, \quad \bar{t}=\frac{\tau_{31}}{\tau_{32}} \frac{t-\tau_{2}}{t-\tau_{1}}, \quad \bar{u}=\frac{\tau_{12}}{\tau_{13}} \frac{u-\tau_{3}}{u-\tau_{2}} .
$$

After this change of variables, the spacetime dependence of the three-point function comes out naturally as a factorized prefactor, and the rest can be combined into a single integral,

$$
\begin{aligned}
C_{\bullet \bullet \bullet} & =\left(\prod_{i=1}^{3} \sqrt{A\left(\Omega_{i}\right)}\right) \times J \\
J & \equiv-\int_{0}^{1} d \bar{s} \int_{0}^{1} d \bar{t} \int_{0}^{1} d \bar{u} \partial_{\bar{u}}\left[f(\bar{u}, \bar{s})^{-\Omega_{1}}\right] \partial_{\bar{s}}\left[f(\bar{s}, \bar{t})^{-\Omega_{2}}\right] \partial_{\bar{t}}\left[f(\bar{t}, \bar{u})^{-\Omega_{3}}\right] .
\end{aligned}
$$

with $f(x, y) \equiv(1-x)^{-1}+y^{-1}-1$. The integral is clearly invariant under any permutation ${ }^{20}$ of $\Omega_{i}$ 's. It is also possible to evaluate the integral $J$ explicitly and express it as an infinite sum, although the result is rather long. See appendix F for the explicit expression.

Let us now expand the result at weak and strong couplings. At weak coupling, one obtains

$$
\begin{aligned}
C \bullet \bullet \bullet & 1+\frac{1}{192 \pi^{2}}\left(\sum_{i}^{3} \hat{\lambda}_{i}^{2}-2 \sum_{i<j} \hat{\lambda}_{i} \hat{\lambda}_{j}\right) \\
& +\frac{\pi^{2}+6 \zeta(3)}{384 \pi^{6}}\left(\sum_{i=1}^{3} \hat{\lambda}_{i}^{3}-\sum_{i<j}\left(\hat{\lambda}_{i}^{2} \hat{\lambda}_{j}+\hat{\lambda}_{i} \hat{\lambda}_{j}^{2}\right)\right)+\frac{\pi^{2}-3-6 \zeta(3)}{192 \pi^{6}} \hat{\lambda}_{1} \hat{\lambda}_{2} \hat{\lambda}_{3}+\mathcal{O}\left(\hat{\lambda}^{4}\right) .
\end{aligned}
$$

One can easily check that the result is consistent with the direct perturbative computation in section 4. Also, by sending one of the effective coupling to zero, one recovers the result in the previous subsection. ${ }^{21}$

To study the leading behavior at strong coupling $\left(\hat{\lambda}_{i} \gg 1\right)$, we use the saddle-point approximation of the integral $J$,

$$
\begin{aligned}
J & =\int_{0}^{1} d \bar{s} \int_{0}^{1} d \bar{t} \int_{0}^{1} d \bar{u} e^{g(\bar{s}, \bar{t}, \bar{u})} \\
g(\bar{s}, \bar{t}, \bar{u}) & =-\Omega_{1} \log f(\bar{u}, \bar{s})-\Omega_{2} \log f(\bar{s}, \bar{t})-\Omega_{3} \log f(\bar{t}, \bar{u})+O(\ln \hat{\lambda}) .
\end{aligned}
$$

\footnotetext{
${ }^{20}$ The invariance under the cyclic permutation can be seen by the permutation of the integrated variables while the reflection invariance can be shown by performing $x \rightarrow 1-x$ to all the integrated variables.

${ }^{21}$ The last term at three loops $\hat{\lambda}_{1} \hat{\lambda}_{2} \hat{\lambda}_{3}$ is truly a new term, which didn't show up in the results in the preceding subsections. It would be interesting to understand it from the integrability point of view.
} 
The subleading term $O(\ln \hat{\lambda})$ can be neglected for the computation of the leading strongcoupling behavior. The saddle-point equation $\partial_{\bar{s}} g=\partial_{\bar{t}} g=\partial_{\bar{u}} g=0$ has two solutions, but one of them is outside the integration region $\bar{s}, \bar{t}, \bar{u} \in[0,1]$ and should be discarded. The one inside the integration region is given by

$$
\left(\bar{s}^{*}, \bar{t}^{*}, \bar{u}^{*}\right)=\left(\frac{\Omega_{1}}{\Omega_{1}+\Omega_{2}}, \frac{\Omega_{2}}{\Omega_{2}+\Omega_{3}}, \frac{\Omega_{3}}{\Omega_{3}+\Omega_{1}}\right) .
$$

Evaluating $g$ at this saddle point and multiplying the prefactors $\prod_{i} \sqrt{A\left(\Omega_{i}\right)}$, we obtain

$$
\ln C \text { •• } \sim \Omega_{1} \log \frac{2 \Omega_{1}}{\Omega_{1}+\Omega_{2}+\Omega_{3}}+\Omega_{2} \log \frac{2 \Omega_{2}}{\Omega_{1}+\Omega_{2}+\Omega_{3}}+\Omega_{3} \log \frac{2 \Omega_{3}}{\Omega_{1}+\Omega_{2}+\Omega_{3}} .
$$

Again the structure of the result suggests that there should be some interpretation in terms of classical string, and it would be extremely interesting if we can re-derive this result from such a perspective.

\section{Discussion}

In this paper, we computed the structure constants on the $1 / 2$ BPS Wilson loop at weak coupling. We first performed the computation at two loops and then resummed the diagrams in the ladders limit. In what follows, we indicate a few possible future directions.

Relation to integrability. As mentioned in the introduction, one of our main motivations was to provide data for the integrability-based approach. In this regard, a natural next step is to reproduce the results in this paper by generalizing the hexagon approach ${ }^{22}$ proposed in [8]. Preliminary investigation suggests that the process involving four (mirror) particles starts to contribute at as early as two loops while the process involving six particles shows up at three loops. Such processes appear much later in the ordinary three-point functions ${ }^{23}$ and it is a great advantage of studying DCO's that we can explore them with the results already available.

From the string worldsheet point of view, the DCO's studied in this paper correspond to the boundary condition changing operators. This viewpoint might provide an alternative approach to the three-point function on the Wilson loop which is based on the form factor expansion of the boundary condition changing operators. See [18] for recent discussions on such form factors in integrable field theories.

Ladders limit. The ladders limit of $\mathcal{N}=4$ SYM provides an invaluable example of the (defect) conformal field theories in higher dimensions, which is exactly calculable but still nontrivial. It would be interesting to explore the properties of the theory further, for instance, by computing non-planar corrections or higher-point functions. Also interesting is to study correlators involving operators outside the loop. We can then start exploring the interplay with the conformal bootstrap [1,2].

\footnotetext{
${ }^{22}$ In a recent paper [17], tree-level three-point functions of non-BPS operators on the Wilson loop were computed using integrability and the open-string version of the hexagon approach was proposed.

${ }^{23}$ At 10 and 14 loops respectively.
} 
Studying the ladders limit would also be useful for the integrability approach itself. To reproduce the result in the ladders limit using integrability, one needs to resum the mirror particles. To our knowledge, it is the only limit where we can predict exact answers after the resummation. ${ }^{24}$ By studying this limit further, we may be able to learn how to perform the resummation in the hexagon approach and make connection with powerful methods developed for the spectrum, such as the thermodynamic Bethe ansatz and the quantum spectral curve.

Furthermore, in this simple set-up, it might be possible to "derive" the hexagon approach from the Feynman diagrams. In the hexagon approach, the structure constants of the DCO's are given in terms of integration of magnon momenta. On the other hand, the perturbative methods in the ladders limit yields integrals over the positions of ends of propagators. Since both integrals are rather simple to analyze, it may be possible to make direct relation between the two. ${ }^{25}$ That would help to clarify the origin of the integrability in $\mathcal{N}=4 \mathrm{SYM}$.

AdS dual of the ladders limit. Yet another interesting problem would be to understand the AdS dual of the ladders limit. A general straight line Wilson loop is dual to a probe worldsheet living in the $A d S_{2}$ subspace of $A d S_{5} \times S^{5}$. In the ladders limit, the worldsheet becomes tensionless while its boundary term remains nontrivial. Although the tensionless limit is in general difficult to analyze, it might be possible to make some progress in this case since one knows the exact answer after the resummation of diagrams. It would be interesting to try to write down the worldsheet action which reproduces the solutions to the Schwinger-Dyson equation.

Recently, certain double-scaling limits of $\mathcal{N}=4 \mathrm{SYM}$ and related theories are advocated as the simplest examples of the integrable gauge/string duality [20-25]. These theories share some features with the ladders limit: first, they have a tensionless limit of the string theory as the bulk dual. Second, in the integrability description, they both arise in the limit where the coupling constant vanishes and the twist diverges. In this sense, the ladders limit may serve as a toy model for such theories. Hopefully understanding the worldsheet description of the ladders limit may be used as a first step towards constructing the bulk dual of such theories.

Lastly, we note that similar resummation of the diagrams was performed recently in $[26,27]$ to determine the cubic coupling of the bulk dual of the SYK model. Although the SYK model is physically quite different from the Wilson loop defect CFT since its bulk dual contains gravity, it might still be interesting to ask if the analysis of the ladders limit has any bearing on this interesting new class of holography.

\footnotetext{
${ }^{24}$ The result at strong coupling also contains information about multi-mirror-particle corrections. However, unlike the ladders limit, it only predicts the leading exponentially dominating contribution.

${ }^{25} \mathrm{~A}$ naive guess is that they are related by the Fourier transformation. It might also be interesting to explore the relation with the Q-functions since in similar set-ups, it was shown that the Schrödinger equation and the Q-functions are related [19].
} 


\section{Acknowledgments}

We acknowledge helpful discussions with Z. Bajnok, D. Correa and N. Drukker. S.K. thanks P. Vieira for a useful conversation. The research of M.K. was supported by a postdoctoral fellowship of the Hungarian Academy of Sciences, a Lendület grant, OTKA 116505 and by the South African Research Chairs Initiative of the Department of Science and Technology and National Research Foundation, whereas the researches of N.K. and T.N. are supported in part by JSPS Research Fellowship for Young Scientists, from the Japan Ministry of Education, Culture, Sports, Science and Technology. The research of S.K. was supported in part by Perimeter Institute for Theoretical Physics. Research at Perimeter Institute is supported by the Government of Canada through the Department of Innovation, Science and Economic Development Canada and by the Province of Ontario through the Ministry of Research, Innovation and Science.

\section{A Basic integrals}

Here we introduce basic integrals following [28], which appear in the perturbative computation. The first integral is the so-called 1-loop conformal integral which is defined by

$$
X_{1234} \equiv \int \frac{d^{4} x_{5}}{x_{15}^{2} x_{25}^{2} x_{35}^{2} x_{45}^{2}},
$$

with $x_{i j}=\left|x_{i}-x_{j}\right|$. This integral can be evaluated explicitly [29] as

$$
X_{1234}=\frac{\pi^{2} \Phi(z, \bar{z})}{x_{13}^{2} x_{24}^{2}}, \quad \Phi(z, \bar{z}) \equiv \frac{2 \operatorname{Li}_{2}(z)-2 \operatorname{Li}_{2}(\bar{z})+\log z \bar{z} \log \frac{1-z}{1-\bar{z}}}{z-\bar{z}},
$$

where $z$ and $\bar{z}$ are the usual conformal cross ratios:

$$
z \bar{z}=\frac{x_{12}^{2} x_{34}^{2}}{x_{13}^{2} x_{24}^{2}}, \quad(1-z)(1-\bar{z})=\frac{x_{14}^{2} x_{23}^{2}}{x_{13}^{2} x_{24}^{2}} .
$$

Another integral which shows up often is the three-point integral given by

$$
Y_{123} \equiv \int \frac{d^{4} x_{5}}{x_{15}^{2} x_{25}^{2} x_{35}^{2}} .
$$

It can be evaluated using $X_{1234}$ as

$$
Y_{123}=\lim _{x_{4} \rightarrow \infty} x_{4}^{2} X_{1234}=\frac{\pi^{2} \Phi\left(z^{\prime}, \bar{z}^{\prime}\right)}{x_{13}^{2}}
$$

with

$$
z^{\prime} \bar{z}^{\prime}=\frac{x_{12}^{2}}{x_{13}^{2}}, \quad\left(1-z^{\prime}\right)\left(1-\bar{z}^{\prime}\right)=\frac{x_{23}^{2}}{x_{13}^{2}} .
$$

When all external points are on a single line, these integrals further simplify to

$$
\begin{aligned}
\left.X_{1234}\right|_{\text {line }} & =-2 \pi^{2}\left(\frac{\log \left(\left|\tau_{12} \tau_{34}\right|\right)}{\tau_{13} \tau_{24} \tau_{14} \tau_{23}}+\frac{\log \left(\left|\tau_{13} \tau_{24}\right|\right)}{\tau_{12} \tau_{34} \tau_{14} \tau_{32}}+\frac{\log \left(\left|\tau_{14} \tau_{23}\right|\right)}{\tau_{12} \tau_{43} \tau_{13} \tau_{42}}\right), \\
\left.Y_{123}\right|_{\text {line }} & =-2 \pi^{2}\left(\frac{\log \left|\tau_{12}\right|}{\tau_{13} \tau_{23}}+\frac{\log \left|\tau_{13}\right|}{\tau_{12} \tau_{32}}+\frac{\log \left|\tau_{23}\right|}{\tau_{21} \tau_{31}}\right),
\end{aligned}
$$

where $\tau_{i}$ 's are the positions of the external points on the line and $\tau_{i j}=\tau_{i}-\tau_{j}$. 


\section{B Vertex and self-energy diagrams for the three-point functions}

In this appendix, we present the detail of the computation of vertex and self-energy diagrams that appear in the three-point functions of DCO's at two loops. As explained in the main text we focus on the terms proportional to $\left(n_{12} \cdot n_{23}-1\right)$.

The contributions from the diagrams listed in figure 9 can be determined in a way similar to the ones in section 3.2. Essentially the only difference is the range of the integration. By changing the range of the integration of the analogous diagrams in section 3.2, one obtains

$$
\begin{aligned}
& \mathrm{T} 1=-\frac{\lambda^{2}\left(n_{12} \cdot n_{23}-1\right)}{4\left(4 \pi^{2}\right)^{3}} \int_{t_{1}^{+}}^{t_{2}^{-}} d \tau_{1} \int_{t_{1}^{+}}^{t_{2}^{-}} d \tau_{2} \int_{t_{2}^{+}}^{t_{3}^{-}} d \tau_{3} \epsilon\left(\tau_{1}-\tau_{2}\right) \partial_{\tau_{1}} Y_{123}, \\
& \mathrm{~T} 2=-\frac{\lambda^{2}\left(n_{12} \cdot n_{23}-1\right)}{4\left(4 \pi^{2}\right)^{3}} \int_{t_{1}^{+}}^{t_{2}^{-}} d \tau_{1} \int_{t_{2}^{+}}^{t_{3}^{-}} d \tau_{2} \int_{t_{2}^{+}}^{t_{3}^{-}} d \tau_{3} \epsilon\left(\tau_{2}-\tau_{3}\right) \partial_{\tau_{2}} Y_{123}, \\
& \mathrm{~T} 3=-\frac{\lambda^{2}\left(n_{12} \cdot n_{23}-1\right)}{4\left(4 \pi^{2}\right)^{3}} \int_{-\infty}^{t_{1}^{-}} d \tau_{1} \int_{t_{1}^{+}}^{t_{2}^{-}} d \tau_{2} \int_{t_{2}^{+}}^{t_{3}^{-}} d \tau_{3}\left(\partial_{\tau_{2}} Y_{123}-\partial_{\tau_{3}} Y_{123}\right), \\
& \mathrm{T} 4=-\frac{\lambda^{2}\left(n_{12} \cdot n_{23}-1\right)}{4\left(4 \pi^{2}\right)^{3}} \int_{t_{1}^{+}}^{t_{2}^{-}} d \tau_{1} \int_{t_{2}^{+}}^{t_{3}^{-}} d \tau_{2} \int_{t_{3}^{+}}^{\infty} d \tau_{3}\left(\partial_{\tau_{1}} Y_{123}-\partial_{\tau_{2}} Y_{123}\right) .
\end{aligned}
$$

As in the computation for the two-point function, we then perform the integration by parts and decompose the integrals into the $\delta$-function terms and the boundary contributions. Here again, the $\delta$-function terms are cancelled by the self-energy diagrams and what remains is

$$
\begin{aligned}
\left.\mathrm{T}\right|_{\text {finite }}=-\frac{\lambda^{2}\left(n_{12} \cdot n_{23}-1\right)}{4(}\left(4 \pi^{2}\right)^{3} & {\left[\int_{t_{1}^{+}}^{t_{2}^{-}} d \tau_{2} \int_{t_{2}^{+}}^{t_{3}^{-}} d \tau_{3}\left(Y_{t_{2}^{-} 23}+Y_{t_{1}^{+} 23}+Y_{t_{3}^{-} 23}+Y_{t_{2}^{+} 23}\right)\right.} \\
& +\int_{-\infty}^{t_{1}^{-}} d \tau_{2} \int_{t_{2}^{+}}^{t_{3}^{-}} d \tau_{3}\left(Y_{t_{2}^{-} 23}-Y_{t_{1}^{+} 23}\right)+\int_{-\infty}^{t_{1}^{-}} d \tau_{2} \int_{t_{1}^{+}}^{t_{2}^{-}} d \tau_{3}\left(Y_{t_{2}^{+} 23}-Y_{t_{3}^{-} 23}\right) \\
& \left.+\int_{t_{2}^{+}}^{t_{3}^{-}} d \tau_{2} \int_{t_{3}^{+}}^{\infty} d \tau_{3}\left(Y_{t_{2}^{-} 23}-Y_{t_{1}^{+} 23}\right)+\int_{t_{1}^{+}}^{t_{2}^{-}} d \tau_{2} \int_{t_{3}^{+}}^{\infty} d \tau_{3}\left(Y_{t_{2}^{+} 23}-Y_{t_{3}^{-} 23}\right)\right] .
\end{aligned}
$$

One can then perform the integral to get

$$
\left.\mathrm{T}\right|_{\text {finite }}=-\left(n_{12} \cdot n_{23}-1\right)\left(\frac{\lambda}{8 \pi^{2}}\right)^{2}\left(3 \zeta(3)+\frac{\pi^{2}}{3} \log \left|\frac{t_{12} t_{23}}{t_{31} \epsilon}\right|\right) .
$$

\section{Excited states and conformal descendants}

In this appendix, we explain the relation between the vertex function $\Gamma_{\epsilon}$ and the Schrödinger equation in $[9,16]$. In particular, we clarify the physical meaning of the wave functions of the Schrödinger equation by showing that they correspond to the three-point functions of DCOs, and that the excited states of the Schrödinger equation correspond to conformal descendants. 
For this purpose, let us quickly review how the Schrödinger equation comes about from the differential equation for $\Gamma_{\epsilon}$ (5.15). To begin with, we rewrite the equation in terms of the "radial coordinate" 26

$$
S=\exp (-x+y), \quad T=\exp (x+y)
$$

to get

$$
\left[-\frac{1}{4}\left(\partial_{x}^{2}-\partial_{y}^{2}\right)-\frac{\hat{\lambda}}{4 \pi^{2}} \frac{1}{(2 \cosh x)^{2}}\right] \Gamma_{\epsilon}=0 .
$$

Physically this rewriting corresponds to considering the theory on $R \times S^{3}: x$ describes the (Euclidean) time difference of the two endpoints while $y$ corresponds to the time of the "center of mass". Then, assuming the form of the solution to be

$$
\Gamma_{\epsilon}=\sum_{N} c_{N} e^{\Omega_{N} y} \Psi_{N}(x)
$$

one can reduce the differential equation (C.2) to the following one-dimensional Schrödinger equation:

$$
\left[-\frac{d^{2}}{d x^{2}}-\frac{\hat{\lambda}}{4 \pi^{2}} \frac{1}{\cosh ^{2} x}\right] \Psi_{N}(x)=-\Omega_{N}^{2} \Psi_{N}(x) .
$$

The Schrödinger equation with this potential (called the Pöschl-Teller potential) has the $\mathrm{SL}(2, \mathbb{R})$ symmetry $^{27}$ and is known to be exactly solvable. This can be seen explicitly by the change of the variable

$$
z=\frac{1}{1+e^{x}}
$$

which maps the problem to the hypergeometric differential equation.

By using the explicit form of $\Gamma_{\epsilon}$ shown in (5.14), one can determine which wave functions appear in the expansion (C.3). The result turns out to be given by a sum of two families of solutions ${ }^{28}$

$$
\Gamma_{\epsilon}=\sum_{n=0}^{\infty} c_{n} e^{\Omega^{(n)} y} \Psi_{n}(x)+\sum_{n=0}^{\infty} \tilde{c}_{n} e^{\tilde{\Omega}^{(n)} y} \tilde{\Psi}_{n}(x)
$$

\footnotetext{
${ }^{26}$ If we instead rewrite the equation in terms of the coordinates $s=S+T$ and $t=S-T$, one arrives at the "conformal quantum mechanics" [30]; the Schrödinger equation with the inverse square potential. This description, however, is not very useful for our purpose and we will not discuss it here.

${ }^{27}$ The difference from the usual conformal quantum mechanics [30] lies in that the "dilatation generator" of the $\mathrm{SL}(2, \mathbb{R})$ is identified not with the Hamiltonian itself but with its square root. See for instance [31].

${ }^{28}$ As in the main text $\Omega$ is defined by
}

$$
\Omega(\hat{\lambda})=\frac{1}{2}\left[-1+\sqrt{1+\frac{\hat{\lambda}}{\pi^{2}}}\right] .
$$


with

$$
\begin{aligned}
\Omega^{(n)} & =\Omega-n, \quad \tilde{\Omega}^{(n)}=-\Omega-n-1, \\
\Psi_{n}(z) & =(z(1-z))^{\frac{\Omega^{(n)}}{2}}{ }_{2} F_{1}\left(\Omega^{(n)}-\Omega, \Omega^{(n)}+\Omega+1,1+\Omega^{(n)} ; z\right), \\
\tilde{\Psi}_{n}(z) & =(z(1-z))^{\frac{\tilde{\Omega}^{(n)}}{2}}{ }_{2} F_{1}\left(\tilde{\Omega}^{(n)}-\Omega, \tilde{\Omega}^{(n)}+\Omega+1,1+\tilde{\Omega}^{(n)} ; z\right) .
\end{aligned}
$$

These solutions have several interesting properties. First, they are the only solutions to (C.4) for which the hypergeometric function reduces to a polynomial. Second, the first family of solutions with $n<\Omega$ decay at $x= \pm \infty$ and correspond to the bound states of the Schrödinger equation (C.4), as discussed in [9]. Note also that, to reconstruct $\Gamma_{\epsilon}$, one needs to include "unphysical solutions" which blow up at $x= \pm \infty$, in addition to such bound state solutions. Although it might seem counter-intuitive, it has natural interpretation in terms of the OPE in the defect CFT as we see below.

To see this, recall that the vertex function is obtained as a limit of the four-point ladder kernel $\Gamma_{\epsilon}(S, T) \equiv K(-S,-\epsilon / 2 \mid \epsilon / 2, T)$. A crucial observation is that the ladder kernel itself can be interpreted as a certain four-point function of (trivial) DCOs,

$$
\Gamma_{\epsilon}(S, T)=K(-S,-\epsilon / 2 \mid \epsilon / 2, T)=\left\langle\mathcal{O}_{1}^{\circ}(-S) \mathcal{O}_{2}^{\circ}(-\epsilon / 2) \mathcal{O}_{3}^{\circ}(\epsilon / 2) \mathcal{O}_{4}^{\circ}(T)\right\rangle,
$$

and the limit $\epsilon \rightarrow 0$ corresponds to the OPE limit where $\mathcal{O}_{2}$ and $\mathcal{O}_{3}$ approach. Using the OPE, one can replace the product of $\mathcal{O}_{2}$ and $\mathcal{O}_{3}$ with an infinite sum, ${ }^{29}$

$$
\mathcal{O}_{2}^{\circ}(-\epsilon / 2) \mathcal{O}_{3}^{\circ}(\epsilon / 2)=\sum_{\tilde{\mathcal{O}}} \epsilon^{\Delta_{\tilde{O}}} c_{23 \tilde{\mathcal{O}}} \tilde{\mathcal{O}}(0) \text {. }
$$

Here the sum on the right hand side is over both primaries and descendants, and $c_{23 \hat{\mathcal{O}}}$ denotes the structure constant. Using this OPE inside the four-point function (C.9), we get the following infinite-sum representation for the vertex function

$$
\Gamma_{\epsilon}(S, T)=\sum_{\tilde{\mathcal{O}}} \epsilon^{\Delta_{\tilde{O}}} c_{23 \tilde{\mathcal{O}}}\left\langle\mathcal{O}_{1}^{\circ}(-S) \tilde{\mathcal{O}}(0) \mathcal{O}_{4}^{\circ}(T)\right\rangle
$$

Let us now compare this sum with the sum over wave functions (C.7). To do so, one has to know the behavior of $\left\langle\mathcal{O}_{1}^{\circ}(-S) \tilde{\mathcal{O}}(0) \mathcal{O}_{4}^{\circ}(T)\right\rangle$ (both for primaries and descendants) and express it in terms of the $x$ and $y$ coordinates. When $\tilde{\mathcal{O}}$ is primary, the behavior of the three-point function is well-known, ${ }^{30}$

$$
\left\langle\mathcal{O}_{1}^{\circ}(-S) \tilde{\mathcal{O}}_{\text {primary }}(0) \mathcal{O}_{4}^{\circ}(T)\right\rangle \propto\left(\frac{S+T}{S T}\right)^{\Delta} .
$$

On the other hand, the behavior for the descendants can be computed by differentiation as

$$
\left\langle\mathcal{O}_{1}^{\circ}(-S) \partial^{n} \tilde{\mathcal{O}}_{\text {primary }}(0) \mathcal{O}_{4}^{\circ}(T)\right\rangle \propto\left(\frac{S+T}{S T}\right)^{\Delta} \sum_{k=0}^{n}\left(\begin{array}{l}
n \\
k
\end{array}\right)(-1)^{k}(\Delta)_{k}(\Delta)_{n-k} \frac{1}{S^{k} T^{n-k}},
$$

\footnotetext{
${ }^{29}$ Here we used the fact that the trivial DCOs have zero conformal dimensions.

${ }^{30}$ For the sake of brevity, below we omit writing the subscript $\tilde{O}$ in $\Delta_{\tilde{O}}$.
} 
with $(x)_{k}$ being the Pochhammer symbol. Re-expressing this in terms of $x$ and $y$, we obtain

$$
\begin{aligned}
(\mathrm{C} .13) & =e^{-(\Delta+n) y}\left(e^{x}+e^{-x}\right)^{\Delta+n} \sum_{k=0}^{n}\left(\begin{array}{l}
n \\
k
\end{array}\right)(-1)^{k}(\Delta)_{k}(\Delta)_{n-k} e^{2 k x}\left(1+e^{-2 x}\right)^{-n} \\
& =e^{-(\Delta+n) y}(z(1-z))^{-\frac{\Delta+n}{2}}\left[\sum_{k=0}^{n}\left(\begin{array}{l}
n \\
k
\end{array}\right)(-1)^{k}(\Delta)_{k}(\Delta)_{n-k} z^{n-k}(1-z)^{k}\right] .
\end{aligned}
$$

In the second line, we further rewrote it in terms of $z=1 /\left(1+e^{x}\right)$. The polynomial in the bracket turns out to be summed into a hypergeometric function ${ }_{2} F_{1}(-n, 1-2 \Delta-n, 1-$ $\Delta-n, z)$. We thus get the expression

$$
\begin{aligned}
& \left\langle\mathcal{O}_{1}^{\circ}(-S) \partial^{n} \tilde{\mathcal{O}}_{\text {primary }}(0) \mathcal{O}_{4}^{\circ}(T)\right\rangle \\
& \propto e^{-(\Delta+n) y}(z(1-z))^{-\frac{\Delta+n}{2}}{ }_{2} F_{1}(-n, 1-2 \Delta-n, 1-\Delta-n, z) .
\end{aligned}
$$

With the identifications $\Delta=-\Omega$ and $\Delta=1+\Omega$, this coincides with $e^{\Omega_{n} y} \Psi_{n}$ and $e^{\tilde{\Omega}_{n} y} \tilde{\Psi}_{n}$ in (C.8) respectively. We can therefore interpret the sum (C.7) really as the OPE expansion and the wave functions are identified with the three-point functions:

$$
\begin{aligned}
& \Gamma_{\epsilon}=\sum_{X=\mathrm{DCO}, \text { shadow }} \sum_{n=0}^{\infty} \epsilon^{\Delta_{X}+n} c_{X, n}\left\langle\mathcal{O}_{1}^{\circ}(-S) \partial^{n} \mathcal{O}_{X}^{\circ}(0) \mathcal{O}_{4}^{\circ}(T)\right\rangle, \\
& \left\langle\mathcal{O}_{1}^{\circ}(-S) \partial^{n} \mathcal{O}_{\mathrm{DCO}}^{\bullet}(0) \mathcal{O}_{4}^{\circ}(T)\right\rangle \quad \leftrightarrow \quad e^{\Omega_{n} y} \Psi_{n} \\
& \left\langle\mathcal{O}_{1}^{\circ}(-S) \partial^{n} \mathcal{O}_{\text {shadow }}^{\bullet}(0) \mathcal{O}_{4}^{\circ}(T)\right\rangle \quad \leftrightarrow \quad e^{\tilde{\Omega}_{n} y} \tilde{\Psi}_{n} .
\end{aligned}
$$

Here $\mathcal{O}_{\mathrm{DCO}}^{\bullet}$ is a nontrivial DCO, which we studied in the main text, and $\mathcal{O}_{\text {shadow }}^{\bullet}$ is its shadow operator, ${ }^{31}$ which has dimension $\Delta_{\text {shadow }}=1-\Delta_{\mathrm{DCO}}=1+\Omega$. This provides a clear physical interpretation of the wave functions for the Schrödinger equation (C.4).

\section{Contribution from the integral of $\Gamma_{U V}$}

In this appendix, we show that, in the $\epsilon \rightarrow 0$ limit, the integrals involving the vertex function $\Gamma_{\epsilon}$ can be approximated by replacing $\Gamma_{\epsilon}$ with its IR counterpart, $\Gamma_{\text {IR }}$. More precisely the goal is to show that the ratio between the contributions from $\Gamma_{\mathrm{UV}}$ and $\Gamma_{\mathrm{IR}}$ is given as follows:

$$
\frac{\int d s \int d t \Gamma_{\mathrm{UV}}(s, t) f(s, t)}{\int d s \int d t \Gamma_{\mathrm{IR}}(s, t) f(s, t)} \leq O(\epsilon \log \epsilon) \stackrel{\epsilon \rightarrow 0}{\rightarrow} 0
$$

Here $f(s, t)$ denotes the rest of the integrand, which may contain other vertex functions, propagators and the ladder kernels $K$.

\footnotetext{
${ }^{31}$ In unitary CFTs, the shadow operators do not usually show up in the spectrum since they are often below the unitarity bound. However, the possibility of having both an operator and its shadow in the spectrum is not totally ruled out. In fact, it is known that some long-range CFTs have such a spectrum.
} 
For this purpose, it is convenient to split the vertex function in a slightly different way as follows:

$$
\begin{aligned}
& \Gamma_{\epsilon}(u)=\tilde{\Gamma}_{\mathrm{IR}}(u)+\tilde{\Gamma}_{\mathrm{UV}}(u), \\
& \tilde{\Gamma}_{\mathrm{IR}}(u)=\frac{A(\Omega)}{(1-u)^{\Omega}} .
\end{aligned}
$$

Since the ratio $\left(\Gamma_{\mathrm{IR}}-\tilde{\Gamma}_{\mathrm{IR}}\right) / \Gamma_{\mathrm{IR}}$ is always of order $O(\epsilon)$ (regardless of their arguments), it is enough to show (D.1) for $\tilde{\Gamma}_{\mathrm{IR}}$ and $\tilde{\Gamma}_{\mathrm{UV}}$.

Now, let us estimate the maximal value of $\tilde{\Gamma}_{\mathrm{UV}}$. In all the examples studied in the main text, the cross ratio $u=\frac{(S-\epsilon / 2)(T-\epsilon / 2)}{(S+\epsilon / 2)(T+\epsilon / 2)}$ takes values in $[0,1-\epsilon / C],{ }^{32}$ with a $O(1)$ positive constant $C$. In this region, the UV vertex $\tilde{\Gamma}_{\mathrm{UV}}$ monotonically decreases in $u$ for $\Omega \leq 1$ while it monotonically increases in $u$ for $\Omega>1.33$ Therefore, the maximal absolute value of the UV vertex is given by

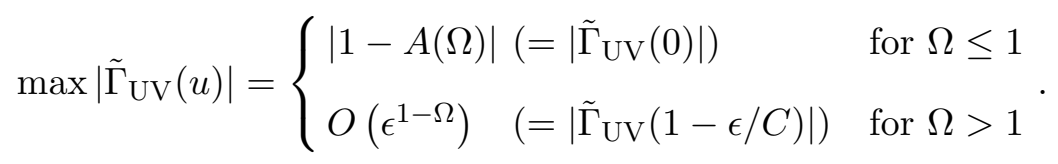

Hence, the integral of $\tilde{\Gamma}_{\text {UV }}$ can be bounded from above as follows:

$$
\int d s \int d t \tilde{\Gamma}_{\mathrm{UV}}(s, t) f(s, t) \leq \frac{\tilde{C}}{\epsilon^{\Omega-1}} \int d s \int d t f(s, t) .
$$

In all the cases encountered in the main text, the integral of $f(s, t)$ can produce at most logarithmic divergences ${ }^{34} \int d s \int d t(s-t)^{-2} \sim \log \epsilon$. We thus have

$$
\int d s \int d t \tilde{\Gamma}_{\mathrm{UV}}(s, t) f(s, t) \leq O\left(\frac{\log \epsilon}{\epsilon^{\Omega-1+|f|}}\right),
$$

where $\epsilon^{-|f|}$ is the singularity contained already in the integrand, $f \sim O\left(\epsilon^{-|f|}\right)$.

On the other hand, since $\tilde{\Gamma}_{\mathrm{IR}} \sim \epsilon^{-\Omega} \times k(s, t)$ with $k(s, t)$ being the $O(1)$ function, we can easily estimate its integral as

$$
\int d s \int d t \tilde{\Gamma}_{\mathrm{IR}}(s, t) f(s, t) \geq O\left(\frac{1}{\epsilon^{\Omega+|f|}}\right) .
$$

Combining (D.5) and (D.6), we get the estimation (D.1) for $\tilde{\Gamma}_{\mathrm{UV}}$ and $\tilde{\Gamma}_{\mathrm{IR}}$.

\section{E Evaluation of the integral (5.35)}

Here we compute the integral

$$
\begin{aligned}
\text { integral } & \equiv \int_{\tau_{1}}^{\tau_{2}} d s \partial_{s} \Gamma^{R}\left(\infty, s-\tau_{1}\right) K\left(s, \tau_{2} \mid \tau_{3}, \infty\right) \\
& =\frac{\sqrt{A(\Omega)}}{\tau_{32}^{\Omega}} \int_{\tau_{1}}^{\tau_{2}} d s \partial_{s}\left[\left(s-\tau_{1}\right)^{\Omega}\right]\left(\tau_{3}-s\right)^{\Omega}{ }_{2} F_{1}\left(-\Omega,-\Omega, 1 ; \frac{\tau_{2}-s}{\tau_{3}-s}\right) .
\end{aligned}
$$

\footnotetext{
${ }^{32} u$ can reach 1 only when $S=T=\infty$. However, we never encounter an integral whose integration regions both extend to infinity.

${ }^{33}$ One can easily verify this by using the definitions of the vertex functions (5.14) and (D.2), and the series expansion of the hypergeometric function.

${ }^{34}$ This inverse square behavior comes from a propagator contained in $f(s, t)$.
} 
As a first step, we perform the change of variables,

$$
x=\frac{s-\tau_{2}}{s-\tau_{3}} \frac{\tau_{1}-\tau_{3}}{\tau_{1}-\tau_{2}},
$$

and use the identity ${ }_{2} F_{1}(a, b, c ; z)=(1-z)^{-a-b+c}{ }_{2} F_{1}(c-a, c-b, c ; z)$ to get

$$
\text { integral }=\Omega \sqrt{A(\Omega)} \tau_{21}^{\Omega}(1-\alpha) \int_{0}^{1} d x(1-x)^{\Omega-1}{ }_{2} F_{1}(\Omega+1, \Omega+1,1 ; \alpha x),
$$

with $\alpha=\tau_{21} / \tau_{32}$. To proceed, we rewrite ${ }_{2} F_{1}$ using the integral representation as

$$
{ }_{2} F_{1}(\Omega+1, \Omega+1,1 ; \alpha x)=\frac{1}{\Gamma(\Omega+1) \Gamma(-\Omega)} \int_{0}^{1} d y y^{\Omega}(1-y)^{-\Omega-1}(1-y \alpha x)^{-\Omega-1} .
$$

One can then perform the $x$ integral to get ${ }^{35}$

$$
\text { integral }=\frac{\sqrt{A(\Omega)} \tau_{21}^{\Omega}(1-\alpha)}{\Gamma(\Omega+1) \Gamma(-\Omega)} \int_{0}^{1} d y y^{\Omega}(1-y)^{-\Omega-1}(1-\alpha y)^{-1} .
$$

This is again a hypergeometric integral and we can compute it as follows:

$$
\text { integral }=\sqrt{A(\Omega)} \tau_{21}^{\Omega}(1-\alpha)_{2} F_{1}(1, \Omega+1,1 ; \alpha) .
$$

Finally, using the identity ${ }_{2} F_{1}(1, \Omega+1,1 ; \alpha)=(1-\alpha)^{-\Omega-1}$, we arrive at

$$
\text { integral }=\frac{\sqrt{A(\Omega)}}{\tau_{21}^{-\Omega} \tau_{32}^{\Omega} \tau_{31}^{-\Omega}} .
$$

\section{F An infinite sum representation for $C_{\bullet}$.}

Here we explicitly evaluate the integral representation for $C_{\text {..॰ }}$ (5.56), and derive an infinitesum representation. First, we perform the following change of variables:

$$
x=\frac{1-\bar{u}}{1-(1-\bar{s}) \bar{u}}, \quad y=1-(1-\bar{s}) \bar{u}, \quad z=\frac{1-\bar{t}}{1-(1-\bar{u}) \bar{t}} .
$$

After taking into account the Jacobian of the transformation, $\frac{y}{(1-x y z)^{2}}$, we get

$$
\begin{aligned}
J= & \prod_{i=1}^{3} \Omega_{i} \int_{0}^{1} d x \int_{0}^{1} d y \int_{0}^{1} d z x^{\Omega_{1}-1} y^{\Omega_{1}} z^{\Omega_{3}-1} \\
& (1-x)^{\Omega_{1}+1}(1-y)^{\Omega_{2}-1}(1-z)^{\Omega_{2}+1}(1-x y)^{-\left(\Omega_{1}+\Omega_{2}-\Omega_{3}+1\right)}(1-y z)^{-\left(\Omega_{2}+1\right)} .
\end{aligned}
$$

The integrals of $x$ and $z$ yield the hypergeometric functions,

$$
\begin{aligned}
J= & \prod_{i=1}^{3} \Omega_{i} \int_{0}^{1} d y y^{\Omega_{1}}(1-y)^{\Omega_{2}-1} \frac{\Gamma\left(\Omega_{3}\right) \Gamma\left(\Omega_{2}+2\right)}{\Gamma\left(\Omega_{2}+\Omega_{3}+2\right)}{ }_{2} F_{1}\left(\Omega_{3}, \Omega_{2}+1, \Omega_{2}+\Omega_{3}+2 ; y\right) \\
& \times \frac{\Gamma\left(\Omega_{1}\right) \Gamma\left(\Omega_{1}+2\right)}{\Gamma\left(2 \Omega_{1}+2\right)}{ }_{2} F_{1}\left(\Omega_{1}, \Omega_{1}+\Omega_{2}-\Omega_{3}+1,2 \Omega_{1}+2 ; y\right) .
\end{aligned}
$$

\footnotetext{
${ }^{35}$ Here we used the integral expression for the hypergeometric function and the identity ${ }_{2} F_{1}(a, 1, a ; z)=$ $(1-z)^{-1}$.
} 
The remaining $y$ integral can be performed using the series expansion of the hypergeometric function and the Euler integral representation for the generalized hypergeometric function:

$$
\begin{aligned}
& { }_{3} F_{2}\left(\begin{array}{c}
\alpha_{1}, \alpha_{2}, \alpha_{3} \\
\beta_{1}, \beta_{2}
\end{array} ; z\right)=\frac{\Gamma\left(\beta_{1}\right) \Gamma\left(\beta_{2}\right)}{\Gamma\left(\alpha_{1}\right) \Gamma\left(\beta_{1}-\alpha_{1}\right) \Gamma\left(\alpha_{2}\right) \Gamma\left(\beta_{2}-\alpha_{2}\right)} \\
& \times \int_{0}^{1} d s \int_{0}^{1} d t s^{\alpha_{1}-1}(1-s)^{\beta_{1}-\alpha_{1}-1} t^{\alpha_{2}-1}(1-t)^{\beta_{2}-\alpha_{2}-1}(1-z s t)^{-\alpha_{3}} .
\end{aligned}
$$

Finally, we obtain the following expression for the structure constant

$$
\begin{aligned}
C \bullet \bullet & \prod_{k=1}^{3} \sqrt{A\left(\Omega_{k}\right)} \frac{\Gamma\left(\Omega_{1}+1\right)^{2} \Gamma\left(\Omega_{1}+2\right) \Gamma\left(\Omega_{2}+1\right) \Gamma\left(\Omega_{2}+2\right) \Gamma\left(\Omega_{3}+1\right)}{\Gamma\left(2 \Omega_{1}+2\right) \Gamma\left(\Omega_{2}+\Omega_{3}+2\right) \Gamma\left(\Omega_{1}+\Omega_{2}+1\right)} \\
& \times \sum_{k=0}^{\infty} \frac{\left(\Omega_{3}\right)_{k}\left(\Omega_{2}+1\right)_{k}}{\left(\Omega_{2}+\Omega_{3}+2\right)_{k} k !} \frac{\left(\Omega_{1}+1\right)_{k}}{\left(\Omega_{1}+\Omega_{2}+1\right)_{k}}{ }_{3} F_{2}\left(\begin{array}{c}
\Omega_{1}, \Omega_{1}+\Omega_{2}-\Omega_{3}+1, \Omega_{1}+k+1 \\
2 \Omega_{1}+2, \Omega_{1}+\Omega_{2}+k+1
\end{array} ; 1\right) .
\end{aligned}
$$

Unlike the integral representation (5.56), this expression is not manifestly symmetric under the permutation of $\Omega_{i}$ 's. One can nevertheless check easily that the expression correctly reproduces $C_{\bullet \bullet \bullet}$ by sending one of $\Omega_{i}$ 's to zero.

Open Access. This article is distributed under the terms of the Creative Commons Attribution License (CC-BY 4.0), which permits any use, distribution and reproduction in any medium, provided the original author(s) and source are credited.

\section{References}

[1] M. Billo, V. Gonçalves, E. Lauria and M. Meineri, Defects in conformal field theory, JHEP 04 (2016) 091 [arXiv:1601.02883] [INSPIRE].

[2] A. Gadde, Conformal constraints on defects, arXiv:1602.06354 [INSPIRE].

[3] N. Drukker, Integrable Wilson loops, JHEP 10 (2013) 135 [arXiv:1203.1617] [INSPIRE].

[4] D. Correa, J. Maldacena and A. Sever, The quark anti-quark potential and the cusp anomalous dimension from a TBA equation, JHEP 08 (2012) 134 [arXiv:1203.1913] [INSPIRE].

[5] N. Gromov and F. Levkovich-Maslyuk, Quantum spectral curve for a cusped Wilson line in $\mathcal{N}=4$ SYM, JHEP 04 (2016) 134 [arXiv:1510.02098] [INSPIRE].

[6] M. Cooke, A. Dekel and N. Drukker, The Wilson loop CFT: insertion dimensions and structure constants from wavy lines, J. Phys. A 50 (2017) 335401 [arXiv:1703.03812] [INSPIRE].

[7] S. Giombi, R. Roiban and A.A. Tseytlin, Half-BPS Wilson loop and $A d S_{2} / C F T_{1}$, Nucl. Phys. B 922 (2017) 499 [arXiv:1706.00756] [INSPIRE].

[8] B. Basso, S. Komatsu and P. Vieira, Structure constants and integrable bootstrap in planar $N=4 S Y M$ theory, arXiv:1505.06745 [INSPIRE]. 
[9] D. Correa, J. Henn, J. Maldacena and A. Sever, The cusp anomalous dimension at three loops and beyond, JHEP 05 (2012) 098 [arXiv:1203.1019] [INSPIRE].

[10] A. Cavaglia, N. Gromov and F. Levkovich-Maslyuk, to appear.

[11] N. Drukker and S. Kawamoto, Small deformations of supersymmetric Wilson loops and open spin-chains, JHEP 07 (2006) 024 [hep-th/0604124] [INSPIRE].

[12] J.K. Erickson, G.W. Semenoff and K. Zarembo, Wilson loops in $N=4$ supersymmetric Yang-Mills theory, Nucl. Phys. B 582 (2000) 155 [hep-th/0003055] [INSPIRE].

[13] N. Drukker and V. Forini, Generalized quark-antiquark potential at weak and strong coupling, JHEP 06 (2011) 131 [arXiv: 1105.5144] [INSPIRE].

[14] N. Drukker and J. Plefka, The structure of n-point functions of chiral primary operators in $N=4$ super Yang-Mills at one-loop, JHEP 04 (2009) 001 [arXiv:0812.3341] [INSPIRE].

[15] S. Giombi, C. Sleight and M. Taronna, Spinning AdS loop diagrams: two point functions, arXiv:1708.08404 [INSPIRE].

[16] J.K. Erickson, G.W. Semenoff, R.J. Szabo and K. Zarembo, Static potential in $N=4$ supersymmetric Yang-Mills theory, Phys. Rev. D 61 (2000) 105006 [hep-th/9911088] [INSPIRE].

[17] M. Kim and N. Kiryu, Structure constants of operators on the Wilson loop from integrability, JHEP 11 (2017) 116 [arXiv:1706. 02989] [INSPIRE].

[18] Z. Bajnok and L. Hollo, On form factors of boundary changing operators, Nucl. Phys. B 905 (2016) 96 [arXiv:1510.08232] [INSPIRE].

[19] N. Gromov and F. Levkovich-Maslyuk, Quark-anti-quark potential in $\mathcal{N}=4$ SYM, JHEP 12 (2016) 122 [arXiv:1601.05679] [InSPIRE].

[20] O. Gurdogan and V. Kazakov, New integrable $4 D$ quantum field theories from strongly deformed planar $\mathcal{N}=4$ supersymmetric Yang-Mills theory, Phys. Rev. Lett. 117 (2016) 201602 [arXiv: 1512.06704] [INSPIRE].

[21] J. Caetano, O. Gurdogan and V. Kazakov, Chiral limit of $N=4 S Y M$ and ABJM and integrable Feynman graphs, arXiv:1612.05895 [INSPIRE].

[22] O. Mamroud and G. Torrents, RG stability of integrable fishnet models, JHEP 06 (2017) 012 [arXiv:1703.04152] [INSPIRE].

[23] D. Chicherin, V. Kazakov, F. Loebbert, D. Müller and D.-l. Zhong, Yangian symmetry for bi-scalar loop amplitudes, arXiv: 1704.01967 [INSPIRE].

[24] N. Gromov et al., Integrability of conformal fishnet theory, arXiv:1706.04167 [INSPIRE].

[25] D. Chicherin et al., Yangian symmetry for fishnet Feynman graphs, arXiv:1708.00007 [INSPIRE].

[26] D.J. Gross and V. Rosenhaus, The bulk dual of SYK: cubic couplings, JHEP 05 (2017) 092 [arXiv: 1702.08016] [INSPIRE].

[27] D.J. Gross and V. Rosenhaus, A line of CFTs: from generalized free fields to SYK, JHEP 07 (2017) 086 [arXiv: 1706.07015] [InSPIRE].

[28] N. Beisert, C. Kristjansen, J. Plefka, G.W. Semenoff and M. Staudacher, BMN correlators and operator mixing in $N=4$ super Yang-Mills theory, Nucl. Phys. B 650 (2003) 125 [hep-th/0208178] [INSPIRE]. 
[29] N.I. Usyukina and A.I. Davydychev, An approach to the evaluation of three and four point ladder diagrams, Phys. Lett. B 298 (1993) 363 [INSPIRE].

[30] C. Chamon, R. Jackiw, S.-Y. Pi and L. Santos, Conformal quantum mechanics as the CFT dual to $A d S_{2}$, Phys. Lett. B 701 (2011) 503 [arXiv:1106.0726] [INSPIRE].

[31] S.H. Dong and R. Lemus, A new dynamical group approach to the modified Poschl-Teller potential, quant-ph/0110157. 